\title{
Channel Modeling of Information Transmission Over Cognitive Interrogator-Sensor Networks
}

\author{
Yifan Chen, Member, IEEE, Wai Lok Woo, and Cheng-Xiang Wang, Senior Member, IEEE
}

\begin{abstract}
This paper looks into the modeling of information transmission over cognitive interrogator-sensor networks (CISNs), which represent a novel and important class of sensor networks deployed for surveillance, tracking, and imaging applications. The crux of the problem is to develop a channel model that allows for the evaluation of the sensing channel capacity and error rate performance in CISNs, for which the sensing link is overlaid by the communication link. First, the layered discrete memoryless channel and finite-state Markov channel models are identified as useful tools to capture the essence of information transfer over the communication and sensing links in CISNs. Two different sensor encoding strategies, namely, 1) the amplify-modulate-and-forward (AMF) and 2) decode-modulate-and-forward (DMF) schemes, are considered. Subsequently, the concepts of double-directional channel capacity azimuth spectrum (CCAS) and Chernoff information azimuth spectrum (CIAS) are proposed to facilitate network-level assessment of the communication and sensing link reliability. A study case considering a typical CISN for environmental monitoring, conditioned on different wireless propagation and sensing conditions, is then presented to demonstrate the steps to derive the various model parameters. Finally, the potential applications of the proposed analytical framework in cognitive sensing, network performance assessment, and simulation are briefly discussed.
\end{abstract}

Index Terms-Channel capacity, Chernoff information, cognitive radar (CR), discrete memoryless channel (DMC), double directional, finite-state Markov channel (FSMC), radio-frequency identification (RFID), wireless sensor network (WSN).

\section{INTRODUCTION}

$\mathbf{C}$ OGNITIVE radar (CR) is an innovative concept for optimizing radar surveillance within a dynamic environment [1]-[5]. A CR has the distinct capabilities of 1) being aware of its outside world by using previous measurements as well

Manuscript received February 25, 2010; revised July 15, 2010, August 28, 2010, and October 13, 2010; accepted October 14, 2010. Date of publication October 25, 2010; date of current version January 20, 2011. The work of C.-X. Wang was supported in part by the Scottish Funding Council for the Joint Research Institute in Signal and Image Processing with the University of Edinburgh, as part of the Edinburgh Research Partnership in Engineering and Mathematics (ERPem), and in part by RCUK for the U.K.-China Science Bridges: R\&D on (B) $4 \mathrm{G}$ Wireless Mobile Communications. The review of this paper was coordinated by Prof. C. P. Oestges.

Y. Chen is with the School of Electrical, Electronic and Computer Engineering, Newcastle University, NE1 7RU Newcastle upon Tyne, U.K., and also with the School of Computer, Electronics and Information, Guangxi University, Nanning 530004, China (e-mail: yifan.chen2@ncl.ac.uk).

W. L. Woo is with the School of Electrical, Electronic and Computer Engineering, Newcastle University, NE1 7RU Newcastle upon Tyne, U.K. (e-mail:w.1.woo@ncl.ac.uk).

C.-X. Wang is with the Joint Research Institute in Signal and Image Processing, School of Engineering and Physical Sciences, Heriot-Watt University, EH14 4AS Edinburgh, U.K. (e-mail: cheng-xiang.wang@hw.ac.uk).

Color versions of one or more of the figures in this paper are available online at http://ieeexplore.ieee.org.

Digital Object Identifier 10.1109/TVT.2010.2089545 as learning through interactions with the environment and 2) intelligently and adaptively customizing the operation of its transceivers in response to the channel variations in real time [1]. The feedback loop from the receiver to the transmitter facilitates such a learning process.

There has also been a large amount of literature dealing with wireless sensor networks (WSNs) over the past few years due to their wide applications in environmental and habitat monitoring, medical diagnostics, disaster management, structural health monitoring, healthcare, etc. [6], [7]. In most of the existing WSNs, a set of nodes measure data from a phenomenon of interest (POI) and then transmit them to a sink, where all data are processed, and global decisions are made. Constrained by limited energy resources and a lack of centralized coordination, a cross-layer design that involves nonconvex nonlinear optimization should be applied to optimize energy consumption in communication and computation [8], and multihop wireless connectivity is usually employed to forward data to and from the sink [9].

Radio-frequency identification (RFID) technology is commonly used to store and retrieve data through RF transmission [9]. An RFID system consists of readers (or interrogators) and tags (or transponders). A tag has a unique identification number and memory that stores additional data. In a typical RFID application, readers communicate with tags in their wireless range and collect information about the objects to which tags are attached, such as manufacturer name, product type, and environmental factors, including temperature, humidity, and so on.

In this paper, we develop a novel network architecture that combines CR, WSN, and RFID and call it a cognitive interrogator-sensor network (CISN) [10]. As illustrated in Fig. 1, the CISN constitutes a dynamic closed feedback loop encompassing the interrogator, environment, and sink. Here, the environment comprises the communication channel (interrogator $\rightarrow$ sensor $\rightarrow$ sink) and the sensing channel (POI $\rightarrow$ sensor). The whole system works as follows.

1) The interrogator sends input signals to activate all sensors in the WSN, which in turn monitor the surroundings to detect a POI.

2) Based on readings collected at sensors, the input data from the interrogator are modulated accordingly and relayed to the sink for central processing and decision making.

3) The interrogator and the sink form a CR system, which continuously learns about the environment through knowledge gained from interactions with the environment and updates the sink with relevant information on the environment. 


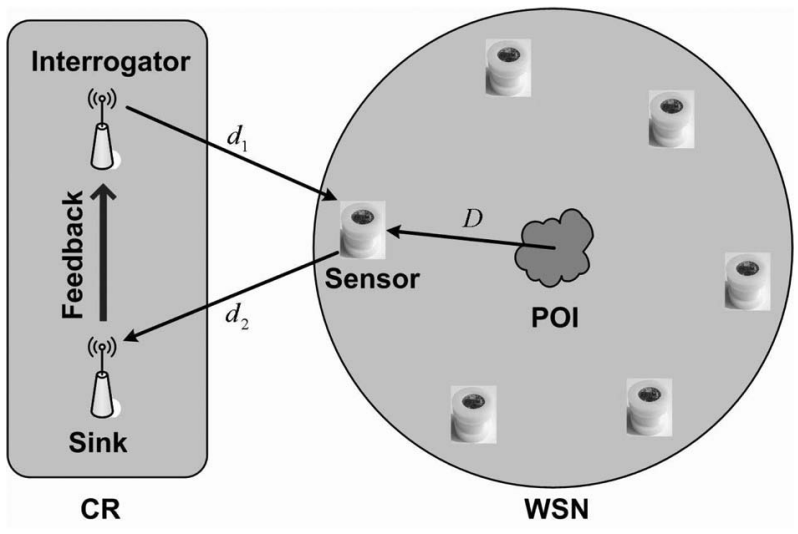

Fig. 1. CISN combining CR, WSN, and RFID technologies.

4) The interrogator adjusts its transmitted signals in a robust and effective manner by taking into account the feedback from the sink. The probing signals may also command the sensors to make adjustments to their sensing and encoding processes.

A CISN conserves power and bandwidth because the network attempts to minimize energy usage by turning off sensor nodes when no communication needs to occur.

\section{A. Related Works}

One fundamental building block of the CISN architecture is a network of sensors with the capability to 1) monitor physical or environmental conditions, 2) store and process information, 3) modulate and demodulate an external "wake-up" signal, and 4) receive and transmit the signal. The first two properties are associated with the traditional WSNs, whereas the last two are related to the RFID, particularly in terms of modulating/ demodulating the wake-up signal. Consequently, these properties require integration of WSN and RFID technologies, which has become feasible in recent years thanks to technological improvements in the miniaturization of sensors, energy savings, and the integration of multiple sensing device within a single node [9], [11]-[23]. In [9], four types of application scenarios for combining RFID and WSNs were discussed: 1) integrating RFID tags with sensors [11]-[19]; 2) integrating tags with WSN nodes and wireless devices [20]; 3) integrating RFID readers with WSN nodes and wireless devices [21]; and 4) a mix of RFID components and WSNs [22], [23].

The CISN is well suited for the first and most investigated type of integration [11]-[19]. The overall functionalities and capacities of the interrogator and the sink in Fig. 1 (except for the feedback loop) are similar to those of an ambient highpower RFID reader, which scans multiple sensor tags in the WSN concurrently and collects information from the network. The sensors in Fig. 1 are essentially RFID tags, which may use the RFID protocols and mechanisms to send tag identifiers as well as to share sensed data. Due to the advance in lowpower electronics, it is now feasible to envisage a low-cost ultralow-power integrated RFID tag that provides powerharvesting, sensing, and actuating capabilities [11]-[13], [15][19]. Such an RFID-tag-and-sensor integration framework may find important applications in bio-monitoring of patients and the elderly [11], [13], [15], [16], cyber-centric monitoring [17], and prognostics and health management [19].

Another important operating block of the CISN is a fully adaptive transmission and reception system with the capability to observe and learn from the environment and adapt its transmission characteristics accordingly. These cognitive sensing features require a feedback loop from the sink to the interrogator [1]-[4], [24], [25], which could be achieved through integration of the CR and RFID reader. The interrogator and the sink can be collocated (i.e., a monostatic radar configuration) and correspond to the transmission and reception modules housed in the same RFID reader circuit. Otherwise, they can be separated by a distance with a feedback connection (i.e., a bistatic arrangement). Nevertheless, little work has been reported in the open literature concerning such integration. Note that incorporating CR into the RFID reader would naturally lead to the integration of CR and WSN through RFID sensor tags.

In brief, the proposed CISN calls for the seamless integration of CR, WSN, and RFID technologies, for which partial integration has already been achieved. To the best of our knowledge, so far, the channel modeling of information transmission over such networks has not explicitly been taken into account to assist in designing an integration architecture. The main objective of the current paper is thus to fill this research gap.

\section{B. Main Contributions of This Paper}

There is a fundamental difference between CISNs and traditional communication networks in terms of information processing. In the former case, we are interested in the differential information between the inputs and outputs of the CR system, which would be correlated with the POI under surveillance. On the other hand, in the latter case, the difference between the inputs and outputs should be zeroed to achieve ideal information delivery. Therefore, it is very important to develop a new theoretical model for the representation and analysis of CISNs. The main contributions of this paper are summarized as follows.

1) We consider two types of information-transmission models: 1) discrete memoryless channel (DMC) and 2) finite-state Markov channel (FSMC) [26]-[28], which are applicable to static and dynamic noisy processes, respectively. Subsequently, the layered DMC and FSMC models are proposed to describe the input-output relationship in a CISN.

2) We introduce two novel sensor encoding strategies for CISN: 1) amplify-modulate-and-forward (AMF) and 2) decode-modulate-and-forward (DMF), which are parallel to the amplify-and-forward and decode-and-forward techniques in cooperative diversity [29]-[32], with the only difference being that an intermediate "modulate" step is included.

3) We develop the double-directional channel capacity azimuth spectrum (CCAS) and the Chernoff information azimuth spectrum (CIAS) to describe the network-scale link quality. 
This paper is organized as follows: In Section II, we establish the general channel modeling methodology for CISNs. Furthermore, a canonical cognitive sensing problem is formulated based on the proposed models. In Section III, a typical CISN for environmental monitoring is presented as a study case, where key model parameters are identified subject to various wireless communication and sensing scenarios. Numerical examples are provided in Section IV based on the discussions in Section III. Section V sheds some light on the potential applications of the proposed methodology. Finally, some concluding remarks are drawn in Section VI.

\section{Cognitive Interrogator-Sensor Network Channel Modeling Methodology}

\section{A. General Principles}

A CISN involves information transmission over two links: 1) the communication link "interrogator $\rightarrow$ sensor $\rightarrow$ sink" and 2) the sensing link "POI $\rightarrow$ sensor," as shown in Fig. 1. To allow for a binary-input-binary-output formulation of these two random processes, we may assume that the sensor is able to encode the continuous-valued POI information into a sequence of binary digits, as demonstrated in the following three examples.

1) Body Sensor Network: There has been a growing interest in the field of body sensor networks for pervasive healthcare applications [13], [33]. Commonly, sensors are placed on the human body to measure specified vital-sign data. These body-worn sensors are simultaneously connected to the gateway that coordinates individual nodes through wireless connectivity. The sensed data may include physiological signals such as electrocardiogram or electroencephalogram. Each sensor can perform standard scalar quantization that maps analog real vital-sign values into discrete real values, which can then be mapped into binary strings using a code book [34].

2) Binary Sensor Network: In the decentralized detection problem, a set of dispersed binary sensor nodes collect information about the POI. Each node receives an analog observation waveform, which is sampled and expressed as a sequence of observations. At each discrete time, the sensor is required to make a binary decision about the POI based on an admissible strategy. The sensor will output 0 if the POI is believed to be absent and output 1 otherwise [35]. Hence, the sensor converts the sensed data into a binary sequence, indicating the presence or absence of the POI.

3) Radar Target Detection: Consider the classical radar detection problem, which assumes a far-field point source target when the radar pulse is narrowband so that the range span of the target is well within a single range cell [36]. Assume that a radar is scanning the surveillance domain to detect a dominant reflecting target. In digital form, the transmitter sends a binary probing sequence $\left(x_{1}, \ldots, x_{K}\right)$, and the receiver observes an output sequence feedback from the environment $\left(y_{1}, \ldots, y_{K}\right)$. For any range cell under surveillance, if an ideal "lossless" reflector is present, then it will either relay the same input data to the receiver (i.e., without phase inversion) or flip all the input bits and then forward them to the receiver (i.e., with phase inversion), depending on its dielectric properties, as compared with the surrounding medium [37]. Effectively, the operation can be expressed as $y_{k}=x_{k} \oplus a_{k} \oplus \eta_{k}(k=1,2, \ldots, K)$, where $a_{k}=0$ or $a_{k}=1$ corresponds to the situation without or with phase inversion, respectively. The term $\eta_{k}$ is the binary channel noise bit, and $\oplus$ is the module- 2 addition. On the other hand, in the absence of a reflector at the range cell under observation, the output $y_{k}$ is dominated by noise, which is given as $y_{k}=\eta_{k}$. Hence, we could interpret the radar target as a virtual sensor, which transforms the information about its location into either an all-one or allzero sequence.

Following from the foregoing discussions, the CISN can be represented using two binary-input-binary-output equations described by

$$
\begin{aligned}
& y_{k}=x_{k} \oplus b_{k} \oplus \eta_{k} \\
& b_{k}=a_{k} \oplus \theta_{k} .
\end{aligned}
$$

Here, $x_{k}$ and $y_{k}$ are the communication link input (data transmitted from the interrogator) and output (data received at the sink), respectively; $a_{k}$ and $b_{k}$ are the sensing link input (ideal POI data without noise corruption) and output (actual POI data used to modulate the probing signals from the interrogator), respectively; $\eta_{k}$ and $\theta_{k}$ are the communication and sensing noise, respectively; and $k$ is the discrete time index. Furthermore, $x_{k}, y_{k}, a_{k}, b_{k}, \eta_{k}, \theta_{k} \in\{0,1\}$, and $\eta_{k}$ and $\theta_{k}$ are two statistically independent processes. These two equations imply that the sensor will behave like a typical relay when $b_{k}=0$, which upon reception of the interrogating signal will forward the same message to the receiver. On the other hand, when $b_{k}=1$, the input data bit will be inverted and then retransmitted to the receiver.

We can broadly categorize the sensor functionality as either nonregenerative or regenerative. In the former case, the sensor amplifies, modulates, and forwards the received signal, whereas in the latter case, the sensor decodes, modulates, and forwards the received signal. These two schemes are parallel to the amplify-and-forward and decode-and-forward techniques in cooperative diversity [29]-[32], with the only difference that an intermediate "modulate" step is included in AMF and DMF to allow the relayed signal to carry information about the POI. Equations (1) and (2) are well suited for an AMF scheme, but for a DMF system, $\eta_{k}$ in (1) should further be decoupled into

$$
\eta_{k}=\eta_{\mathrm{B}, k} \oplus \eta_{\mathrm{F}, k}
$$

In the preceding equation, $\eta_{\mathrm{B}, k}$ and $\eta_{\mathrm{F}, k}$ are associated with the noise processes of the backward channel (interrogator $\rightarrow$ sensor) and the forward channel (sensor $\rightarrow$ sink), respectively. Nevertheless, we may establish an equivalent AMF channel from the underlying DMF channel by applying the following theorem on cascading two independent binary symmetric channels (BSCs).

Theorem 1-Cascading of Two BSCs: If $\eta_{\mathrm{B}, k}$ and $\eta_{\mathrm{F}, k}$ can be described by two independent BSCs with raw error 


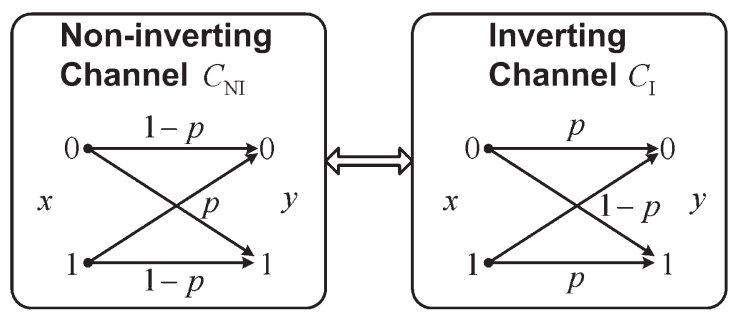

(a)

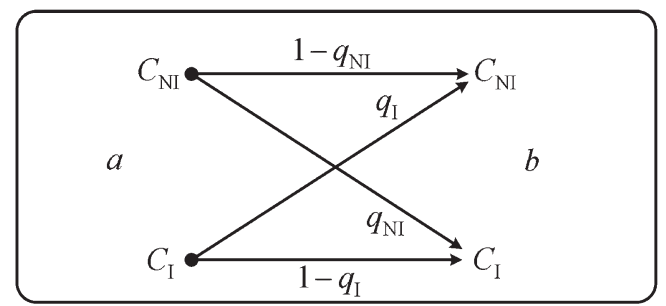

(b)

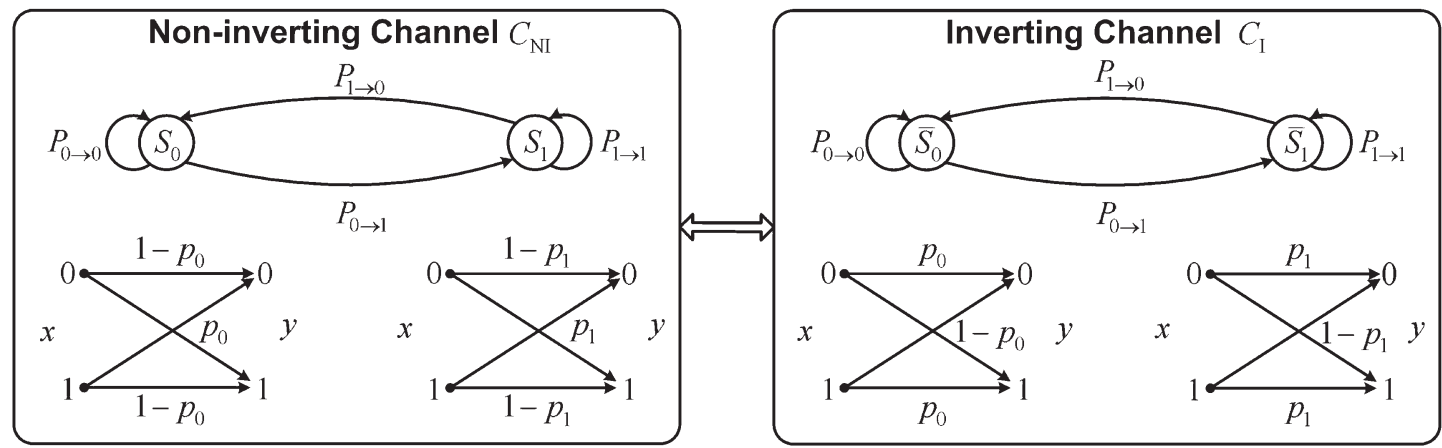

(c)

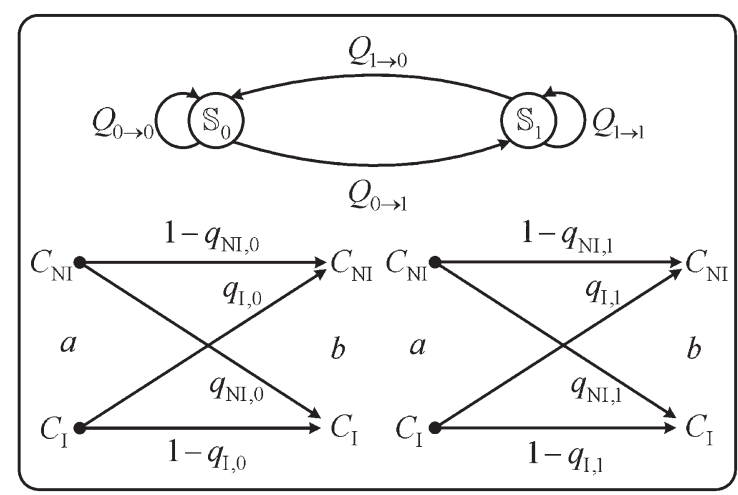

(d)

Fig. 2. Layered DMC and FSMC models. (a) BSCs for a time-invariant communication link. (b) DMC for a time-invariant sensing link. (c) FSMCs for a time-varying communication link. (d) FSMC for a time-varying sensing link.

probabilities $p_{\mathrm{B}}$ and $p_{\mathrm{F}}$, respectively, then $\eta_{k}=\eta_{\mathrm{B}, k} \oplus \eta_{\mathrm{F}, k}$ is equivalent to a single $\mathrm{BSC}$ with error probability $p=p_{\mathrm{B}}+$ $p_{\mathrm{F}}-2 p_{\mathrm{B}} p_{\mathrm{F}}$.

Proof: The probability that $\eta_{k}=1$ is given by

$$
\begin{aligned}
\operatorname{Pr}\left\{\eta_{k}=1\right\}= & \operatorname{Pr}\left\{\eta_{\mathrm{B}, k}=0 ; \eta_{\mathrm{F}, k}=1\right\}+\operatorname{Pr}\left\{\eta_{\mathrm{B}, k}=1 ; \eta_{\mathrm{F}, k}=0\right\} \\
& \stackrel{(a)}{=} \operatorname{Pr}\left\{\eta_{\mathrm{B}, k}=0\right\} \operatorname{Pr}\left\{\eta_{\mathrm{F}, \mathrm{k}}=1\right\} \\
& \quad+\operatorname{Pr}\left\{\eta_{\mathrm{B}, k}=1\right\} \operatorname{Pr}\left\{\eta_{\mathrm{F}, \mathrm{k}}=0\right\} \\
& \stackrel{(b)}{=}\left(1-p_{\mathrm{B}}\right) p_{\mathrm{F}}+p_{\mathrm{B}}\left(1-p_{\mathrm{F}}\right) \\
= & p_{\mathrm{B}}+p_{\mathrm{F}}-2 p_{\mathrm{B}} p_{\mathrm{F}}
\end{aligned}
$$

where $\operatorname{Pr}\{\cdot\}$ denotes probability. The equality in $(a)$ follows the assumption that $\eta_{\mathrm{B}, k}$ and $\eta_{\mathrm{F}, k}$ are two independent random processes. Furthermore, the result in $(b)$ is due to the fact that both $\eta_{\mathrm{B}, k}$ and $\eta_{\mathrm{F}, k}$ are BSCs. From (4), we can see that $\eta_{k}$ is also a BSC with crossover probability $p_{\mathrm{B}}+p_{\mathrm{F}}-2 p_{\mathrm{B}} p_{\mathrm{F}}$. This completes the proof.
In the following analysis, unless otherwise stated, we will use a generic $\eta_{k}$ to represent the noise process of the communication link for both AMF and DMF systems.

\section{B. Layered DMC and FSMC Models}

1) Layered DMC Model: Consider the scenario when both (1) and (2) represent time-invariant processes with $\eta_{k}$ and $\theta_{k}$ being two binary random variables. If the underlying continuous-time noise in the communication and sensing channels is additive white Gaussian noise (AWGN), then (1) and (2) could be described by an overlay DMC model. First, two different situations can be identified from (1). When $b_{k}=0$, (1) reduces to a standard discrete memoryless BSC with crossover probability $p$ (i.e., $\operatorname{Pr}\left\{\eta_{k}=1\right\}=p$ ) [34], [38]. Alternatively, $b_{k}=1$ yields a BSC with crossover probability $1-p$, which can easily be verified by observing that the error probability $\operatorname{Pr}\left\{y_{k} \oplus x_{k}=1\right\} \equiv \operatorname{Pr}\left\{b_{k} \oplus \eta_{k}=1\right\}=\operatorname{Pr}\left\{\eta_{k}=\right.$ $0\}=1-p$. In the following, we will refer to these two situations as the noninverting $\left(C_{\mathrm{NI}}\right)$ and inverting $\left(C_{\mathrm{I}}\right)$ channels, respectively, as depicted in Fig. 2(a). 
Subsequently, the underlying law that governs the transition between $C_{\mathrm{NI}}$ and $C_{\mathrm{I}}$ is given by (2), which could be modeled using a DMC with two different error probabilities $q_{\mathrm{NI}}$ and $q_{\mathrm{I}}$, as shown in Fig. 2(b) (i.e., $\operatorname{Pr}\left\{\theta_{k}=1\right\}=q_{\mathrm{NI}}$ if $a_{k}=0$ and $\operatorname{Pr}\left\{\theta_{k}=1\right\}=q_{\mathrm{I}}$ if $a_{k}=1$ ). The main reason to use an inputdependent DMC instead of a BSC is due to the specific features associated with a sensing link, which will be made clear in Section III-A.

In brief, a layered DMC model comprising the DMC in Fig. 2(b) overlaid by the two BSCs in Fig. 2(a) can be employed to characterize the input-output relationship in a CISN if both the wireless propagation channel and the POI are time invariant.

2) Layered DMC/FSMC Model: If (1) is a time-varying process and (2) is time invariant, then we can still apply the DMC in Fig. 2(b) to model (2). However, two FSMCs, as shown in Fig. 2(c), should be used to characterize the timevarying fading channel in (1). When $b_{k}=0$, we can define a standard FSMC for the noninverting channel $C_{\mathrm{NI}}$ [26], [39]. Each state is associated with a BSC. For example, the crossover probabilities for the two states of $C_{\mathrm{NI}}$ in Fig. 2(c), i.e., $S_{0}$ and $S_{1}$, are $p_{0}$ (i.e., $\left.\operatorname{Pr}\left\{\eta_{k}=1\right\}=p_{0}\right)$ and $p_{1}$ (i.e., $\operatorname{Pr}\left\{\eta_{k}=1\right\}=$ $\left.p_{1}\right)$, respectively. The analytical expressions for states, state transition probabilities, and error probabilities in each state conditioned on the time-varying communication link process follow the widely used techniques in [27]. On the other hand, $b_{k}=1$ results in a similarly structured FSMC with "inverting" states $\bar{S}_{0}$ and $\bar{S}_{1}$, where the crossover probabilities are given by $1-p_{0}$ and $1-p_{1}$, respectively. This phenomenon can readily be proved following the same approach in Section II-B1. This type of Markov chain gives rise to the inverting channel $C_{\mathrm{I}}$. Hence, a layered FSMC-DMC model that comprises the DMC in Fig. 2(b) overlaid by the two FSMCs in Fig. 2(c) should be employed to describe the input-output relationship in a CISN.

Conversely, if (1) is time invariant but (2) is time varying, we can make use of the two BSCs in Fig. 2(a) to describe (1). However, an FSMC, as illustrated in Fig. 2(d), should be considered for the time-varying process in (2) [39]. Each state in Fig. 2(d) is associated with a DMC. For example, the error probabilities for state $\mathbb{S}_{0}$ in Fig. $2(\mathrm{~d})$ are given as $q_{\mathrm{NI}, 0}$ (i.e., $\operatorname{Pr}\left\{\theta_{k}=1\right\}=q_{\mathrm{NI}, 0}$ if $a_{k}=0$ ) and $q_{\mathrm{I}, 0}$ (i.e., $\operatorname{Pr}\left\{\theta_{k}=1\right\}=$ $q_{\mathrm{I}, 0}$ if $a_{k}=1$ ). Moreover, all the other model parameters can be derived following the approach similar to that in [27]. In summary, a layered DMC-FSMC model that comprises the FSMC in Fig. 2(d) overlaid by the two BSCs in Fig. 2(a) should be used to explain the input-output relationship in a CISN in this situation.

3) Layered FSMC Model: Finally, the most complex scenario occurs when both (1) and (2) are time-varying processes. In such a case, a layered FSMC model that consists of the Markov chain in Fig. 2(d) overlaid by the Markov chains in Fig. 2(c) is appropriate for synthesizing the two input-output equations in (1) and (2), respectively.

\section{Double-Directional CCAS and CIAS}

Analogous to the fact that the signal power is flowing through a physical multipath channel, the relevant substance that is flowing through a virtual CISN is the information delivered

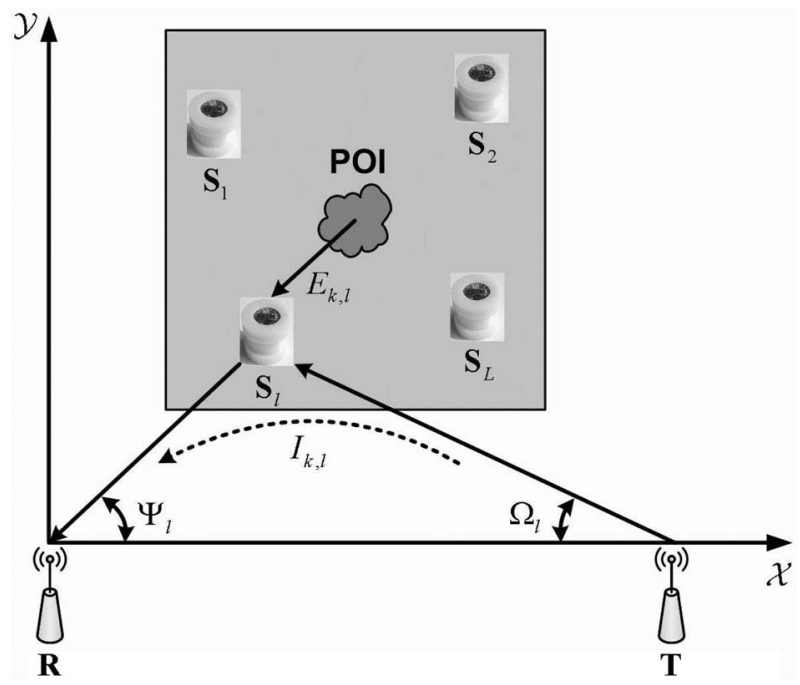

Fig. 3. Double-directional CCAS and CIAS.

from the interrogator to the sink. The sensors serve the role of information propagation medium, parallel to the role of scattering objects in physical channels as power reradiators [40]. Inspired by this noteworthy similarity, we consider a doubledirectional description of the network, parallel to the doubledirectional characterization of the propagation channel [41]. Consider a CISN consisting of $L+2$ nodes: an interrogator $\mathbf{T}$, a sink $\mathbf{R}$, and $L$ noncollocated sensors $\mathbf{S}_{1}, \mathbf{S}_{2}, \ldots, \mathbf{S}_{L}$, which are arbitrarily distributed in a given region, as illustrated in Fig. 3. Multiple information pipelines are laid via the $L$ links $\mathbf{T} \rightarrow \mathbf{S}_{1} \rightarrow \mathbf{R}, \ldots, \mathbf{T} \rightarrow \mathbf{S}_{L} \rightarrow \mathbf{R}$. Furthermore, the intersection of each angle-of-departure (AOD) $\Omega_{l}$ and each angle-ofarrival (AOA) $\Psi_{l}$ uniquely determines the location of sensor $\mathbf{S}_{l}$. Therefore, at discrete time $k$, the information flows in the network are distributed in the AOD-AOA domain as

$$
I_{k}(\Omega, \Psi)=\sum_{l=1}^{L} I_{k, l} \delta\left(\Omega-\Omega_{l}\right) \delta\left(\Psi-\Psi_{l}\right)
$$

where $I_{k}(\Omega, \Psi)$ is defined as the double-directional CCAS, and $I_{k, l}$ is the channel capacity at time $k$ for the communication link via sensor $\mathbf{S}_{l}$. The terminology double directional is adopted according to [41]. If the connection $\mathbf{T} \rightarrow \mathbf{S}_{l} \rightarrow \mathbf{R}$ follows a BSC with error probability $p_{k, l}$, then the channel capacity can be calculated to be [38]

$$
I_{k, l}=1+p_{k, l} \log _{2} p_{k, l}+\left(1-p_{k, l}\right) \log _{2}\left(1-p_{k, l}\right) .
$$

Note that this value is the same for both noninverting and inverting channels.

Equation (5) provides a network-level assessment of the communication link reliability. In a similar manner, we may devise another type of double-directional spectrum to evaluate the sensing link performance. Assume that the sensing link for sensor $\mathbf{S}_{l}$ at time $k$ can be characterized using the DMC in Fig. 2(b) with error probabilities $q_{\mathrm{NI}, k, l}$ and $q_{\mathrm{I}, k, l}$ for $C_{\mathrm{NI}}$ and $C_{\mathrm{I}}$, respectively. Essentially, this is equivalent to a hypothesis testing problem and can be restated as follows. 
Problem 1: At time $k$, sensor $\mathbf{S}_{l}$ decides between two alternative hypotheses $H_{0}$ ("Channel is $C_{\mathrm{NI}}$ ") and $H_{1}$ ("Channel is $C_{\mathrm{I}}$ ") subject to the probability of detection $\operatorname{Pr}\left\{b_{k, l}=1 \mid H_{1}\right\}=$ $1-q_{\mathrm{I}, k, l}$ and the probability of false alarm $\operatorname{Pr}\left\{b_{k, l}=1 \mid H_{0}\right\}=$ $q_{\mathrm{NI}, k, l}$, where $b_{k, l}$ is the output of the sensing link [cf. (2)]. The best achievable error exponent for this problem is given by the Chernoff information [38] as

$$
\begin{array}{r}
E_{k, l}=-\min _{0 \leq \epsilon \leq 1} \log _{2}\left\{\sum_{b_{k, l} \in\{0,1\}}\left[\operatorname{Pr}\left\{b_{k, l} \mid H_{0}\right\}\right]^{\epsilon}\left[\operatorname{Pr}\left\{b_{k, l} \mid H_{1}\right\}\right]^{1-\epsilon}\right\} \\
=-\min _{0 \leq \epsilon \leq 1} \log _{2}\left\{\left[\operatorname{Pr}\left\{0 \mid H_{0}\right\}\right]^{\epsilon}\left[\operatorname{Pr}\left\{0 \mid H_{1}\right\}\right]^{1-\epsilon}\right. \\
\left.+\left[\operatorname{Pr}\left\{1 \mid H_{0}\right\}\right]^{\epsilon}\left[\operatorname{Pr}\left\{1 \mid H_{1}\right\}\right]^{1-\epsilon}\right\} \\
=-\min _{0 \leq \epsilon \leq 1} \log _{2}\left[\left(1-q_{\mathrm{NI}, k, l}\right)^{\epsilon} q_{\mathrm{I}, k, l}^{1-\epsilon}+q_{\mathrm{NI}, k, l}^{\epsilon}\left(1-q_{\mathrm{I}, k, l}\right)^{1-\epsilon}\right] .
\end{array}
$$

For simplicity, we will consider the Bhattacharyya distance in this paper, which is a special case of Chernoff information [38], as given by $E_{k, l}=-\log _{2}\left[\left(1-q_{\mathrm{NI}, k, l}\right)^{1 / 2} q_{\mathrm{I}, k, l}^{1 / 2}+\right.$ $\left.q_{\mathrm{NI}, k, l}^{1 / 2}\left(1-q_{\mathrm{I}, k, l}\right)^{1 / 2}\right]$.

Subsequently, we can define the double-direction CIAS as

$$
E_{k}(\Omega, \Psi)=\sum_{l=1}^{L} E_{k, l} \delta\left(\Omega-\Omega_{l}\right) \delta\left(\Psi-\Psi_{l}\right)
$$

which offers a network-scale evaluation of the sensing link quality.

\section{Canonical Cognitive Sensing Problem}

Let us consider time-varying communication and sensing channels. We will use the superscript ${ }^{\hat{k}}$ to indicate the slow time index for symbol vectors or system-level parameters (e.g., system operational modes or performance measures) at each round of global adaptation. This should be distinguished from the subscript ${ }_{k}$ that represents the fast time index for elements collected in symbol vectors. Furthermore, the subscripts $\mathbf{T}, \mathbf{S}$, and $\mathbf{R}$ will be used to denote the quantities associated with the interrogator, sensor, and sink, respectively. Subsequently, the following variables at discrete time $\hat{k}$ for a CISN can be defined:

1) $\mathcal{B}_{\mathrm{T}}^{\hat{k}}$ : beamforming strategy at the interrogator;

2) $\mathcal{B}_{\mathrm{R}}^{\hat{k}}$ : beamforming strategy at the sink;

3) $X^{\hat{k}} \triangleq\left(x_{1}^{\hat{k}}, x_{2}^{\hat{k}}, \ldots, x_{K}^{\hat{k}}\right)$ : input binary sequence transmitted from the interrogator, where $K$ is the length of the sequence at time $\hat{k}$;

4) $Y^{\hat{k}} \triangleq\left(y_{1}^{\hat{k}}, y_{2}^{\hat{k}}, \ldots, y_{K}^{\hat{k}}\right)$ : output binary sequence received at the sink;

5) $\mathcal{C}^{\hat{k}} \triangleq\left\{\mathcal{C}_{1}^{\hat{k}}, \mathcal{C}_{2}^{\hat{k}}, \ldots, \mathcal{C}_{L}^{\hat{k}}\right\}$ : coding strategies of sensors (i.e., mapping of continuous-valued sensing data into binary sequences) administered by the interrogator, where $\mathcal{C}_{l}^{\hat{k}}(l=1,2, \ldots, L)$ : the admissible strategy at sensor $\mathbf{S}_{l}$;
6) $\mathbf{L}^{\hat{k}} \triangleq\left\{\mathbf{l}_{1}^{\hat{k}}, \mathbf{l}_{2}^{\hat{k}}, \ldots, \mathbf{l}_{L}^{\hat{k}}\right\}$ : location estimate of sensors at the sink, where $\mathbf{l}_{l}^{\hat{k}}(l=1,2, \ldots, L)$ denotes the location estimate of sensor $\mathbf{S}_{l}$;

7) $\mathbf{P}^{\hat{k}} \triangleq\left\{\mathbf{p}_{1}^{\hat{k}}, \mathbf{p}_{2}^{\hat{k}}, \ldots, \mathbf{p}_{L}^{\hat{k}}\right\}$ : estimated layered FSMC model parameters [cf. Fig. 2(c) and (d)] for all the sensors, where $\mathbf{p}_{l}^{\hat{k}}(l=1,2, \ldots, L)$ denotes the estimated parameter set for sensor $\mathbf{S}_{l}$.

Subsequently, a canonical cognitive sensing problem can be formulated as follows.

Problem 2:

$$
\begin{array}{ll}
\text { Given } & \mathcal{B}_{\mathbf{T}}^{\hat{k}}, \mathcal{B}_{\mathbf{R}}^{\hat{k}}, X^{\hat{k}}, Y^{\hat{k}}, \mathcal{C}^{\hat{k}}, \mathbf{L}^{\hat{k}}, \mathbf{P}^{\hat{k}} \\
\text { Optimize } & \mathcal{M}^{\hat{k}+1} \text { at time } \hat{k}+1 \\
\text { Subject to } & \mathcal{B}_{\mathbf{T}}^{\hat{k}+1} \in \mathbb{B}_{\mathbf{T}}, \mathcal{B}_{\mathbf{R}}^{\hat{k}+1} \in \mathbb{B}_{\mathbf{R}}, X^{\hat{k}+1} \in \mathbb{X}, \mathcal{C}^{\hat{k}+1} \in \mathbb{C} .
\end{array}
$$

The optimization criteria $\mathcal{M}^{\hat{k}+1}$ represents a specific system performance measure under consideration, which may be the communication channel capacity, sensing error rate, sensing waveform fidelity, etc. $\mathbb{B}_{\mathbf{T}}, \mathbb{B}_{\mathbf{R}}, \mathbb{X}$, and $\mathbb{C}$ are the admissible sets of relevant strategies or system parameters. Note that the foregoing canonical problem can easily be applied to other types of communication or sensing channels.

\section{Derivation of Model Parameters in a Typical COGNITIVE INTERROGATOR-SENSOR NETWORK}

\section{A. Time-Invariant Wireless Channel and POI}

When both the wireless channel and the POI are time invariant, the information transfer over a CISN can be characterized by using the layered DMC model presented in Section II-B1.

The simplest case of a time-invariant communication link is an AWGN channel [42]. It is assumed that the average power transmitted by the interrogator and the sensor is $\mathcal{P}_{\mathbf{T}}^{\mathrm{t}}$ and $\mathcal{P}_{\mathbf{S}}^{\mathrm{t}}$, respectively, where the superscript ${ }^{\mathrm{t}}$ denotes the transmitted signal. For simplicity, we further assume that both the sensor and sink receiver chains have identical noise properties and hence the same AWGN power. Nevertheless, the following analysis is also applicable to systems with unequal sensor and sink noise power.

1) AMF System: For an AMF system, the wireless channel consists of two portions: the 1) backward (interrogator $\rightarrow$ sensor) and 2) forward (sensor $\rightarrow$ sink) links, as depicted in Fig. 1. The following received signal at the sink $y_{\mathbf{R}}(t)$ comprises three components, as demonstrated in [30]:

$$
y_{\mathbf{R}}(t)=h_{\mathrm{F}} h_{\mathrm{B}} x_{\mathbf{S}}(t)+h_{\mathrm{F}} n(t)+n(t)
$$

where $n(t)$ is the AWGN waveform, and $x_{\mathbf{S}}(t)$ is the unitvariance modulated signal sent by the sensor. The two variables $h_{\mathrm{B}}$ and $h_{\mathrm{F}}$ correspond to the backward and forward channels, respectively, which can be calculated as [5]

$$
h_{\mathrm{B}}=\sqrt{\mathcal{P}_{\mathbf{T}}^{\mathrm{t}}} A d_{1}^{-\frac{\kappa}{2}} \quad \text { and } \quad h_{\mathrm{F}}=\sqrt{G_{\mathbf{S}}} A d_{2}^{-\frac{\kappa}{2}} .
$$

In (10), $A$ is a constant, $G_{\mathbf{S}}$ is the power amplification factor of the sensor, and $\kappa$ is the path loss exponent. $d_{1}$ and $d_{2}$ 
are the propagation distances for the backward and forward connections, respectively, as shown in Fig. 1. For simplicity, we will consider a fixed gain sensor (parallel to a fixed gain relay in cooperative diversity systems [30], [31]) with

$$
G_{\mathbf{S}}=\frac{\mathcal{P}_{\mathbf{S}}^{\mathrm{t}}}{\mathrm{E}\left\{\mathcal{P}_{\mathbf{S}}^{\mathrm{r}}\right\}}=\frac{\mathcal{P}_{\mathbf{S}}^{\mathrm{t}}}{\mathcal{P}_{\mathbf{T}}^{\mathrm{t}} A^{2} d_{1}^{-\kappa}+\sigma_{n}^{2}} .
$$

$\mathrm{E}\{\cdot\}$ is the statistical expectation operation, and $\mathcal{P}_{\mathrm{S}}^{\mathrm{r}}$ is the power received at the sensor, where the superscript ${ }^{\mathrm{r}}$ denotes the received signal. $\sigma_{n}^{2}$ is the power of the AWGN in the backward link. Substituting (10) and (11) into (9) yields

$$
\begin{aligned}
y_{\mathbf{R}}(t)= & h_{\mathrm{F}} h_{\mathrm{B}} x_{\mathbf{S}}(t)+\left(h_{\mathrm{F}}+1\right) n(t) \\
= & \sqrt{\frac{\mathcal{P}_{\mathbf{S}}^{\mathrm{t}}}{1+\sigma_{n}^{2} /\left(\mathcal{P}_{\mathbf{T}}^{\mathrm{t}} A^{2} d_{1}^{-\kappa}\right)}} A d_{2}^{-\frac{\kappa}{2}} x_{\mathbf{S}}(t) \\
& +\left(\sqrt{\frac{\mathcal{P}_{\mathbf{S}}^{\mathrm{t}}}{\mathcal{P}_{\mathbf{T}}^{\mathrm{t}} A^{2} d_{1}^{-\kappa}+\sigma_{n}^{2}}} A d_{2}^{-\frac{\kappa}{2}}+1\right) n(t)
\end{aligned}
$$

where the first term is the desired signal, whereas the second term is the overall AWGN.

Subsequently, $C_{\mathrm{NI}}$ and $C_{\mathrm{I}}$ are defined by the BSCs in Fig. 2(a). For binary phase-shift keying (BPSK), the crossover probability for $C_{\mathrm{NI}}$ is given by [42]

$$
\begin{aligned}
p & =\mathcal{Q}\left(\sqrt{2\left(\frac{h_{\mathrm{F}} h_{\mathrm{B}}}{h_{\mathrm{F}}+1}\right)^{2} \gamma}\right) \\
& =\mathcal{Q}\left(\sqrt{\frac{2 \mathcal{P}_{\mathbf{S}}^{\mathrm{t}} A^{2} d_{2}^{-\kappa} \gamma}{\left[1+\frac{\sigma_{n}^{2}}{\mathcal{P}_{\mathbf{T}}^{\mathrm{t}} A^{2} d_{1}^{-\kappa}}\right]\left[\sqrt{\frac{\mathcal{P}_{\mathrm{S}}^{\mathrm{t}} A^{2} d_{2}^{-\kappa} / \sigma_{n}^{2}}{\mathcal{P}_{\mathbf{T}}^{\mathrm{t}} A^{2} d_{1}^{-\kappa} / \sigma_{n}^{2}+1}}+1\right]^{2}}}\right) .
\end{aligned}
$$

The $\mathcal{Q}(\cdot)$ function is the complementary cumulative normal distribution. $\gamma=E_{b} / N_{0}$, where $E_{b}$ is the average energy per bit for the unit-variance signal $x_{\mathbf{S}}(t)$, and $N_{0}$ is the noise spectral density of $n(t)$.

2) DMF System: For a DMF system, the signal received at the sensor is written as

$$
y_{\mathbf{S}}(t)=h_{\mathrm{B}} x_{\mathbf{T}}(t)+n(t)
$$

where $x_{\mathbf{T}}(t)$ is the unit-variance waveform sent by the interrogator with the same average energy per bit as that of $x_{\mathbf{S}}(t)$, and $h_{\mathrm{B}}$ is given in (10). For BPSK, the backward channel can be described by a BSC with error probability

$$
p_{\mathrm{B}}=\mathcal{Q}\left(\sqrt{2 h_{\mathrm{B}}^{2} \gamma}\right)=\mathcal{Q}\left(\sqrt{2 \mathcal{P}_{\mathbf{T}}^{\mathrm{t}} A^{2} d_{1}^{-\kappa} \gamma}\right) .
$$

Furthermore, the signal received at the sink from the sensor (after decoding and modulating) is

$$
y_{\mathbf{R}}(t)=h_{\mathrm{F}}^{\prime} x_{\mathbf{S}}(t)+n(t)
$$

where $h_{\mathrm{F}}^{\prime}=\sqrt{\mathcal{P}_{\mathrm{S}}^{\mathrm{t}}} A d_{2}^{-(\kappa / 2)}$. For BPSK, the forward channel can be described by a BSC with crossover probability as

$$
p_{\mathrm{F}}=\mathcal{Q}\left(\sqrt{2\left(h_{\mathrm{F}}^{\prime}\right)^{2} \gamma}\right)=\mathcal{Q}\left(\sqrt{2 \mathcal{P}_{\mathrm{S}}^{\mathrm{t}} A^{2} d_{2}^{-\kappa} \gamma}\right) .
$$

Substituting (15) and (17) into (4) gives the error probability of the equivalent single BSC for the DMF system.

3) POI: The simplest model for the POI is a time-invariant isotropic signal source with path loss factor $\beta$ [43], which depends on the type of signal considered (chemical contamination, sound, radioactive radiation, etc.). Thus, the received signal strength at the sensor $\rho_{k}$ after appropriate sampling and processing is

$$
\rho_{k}= \begin{cases}w_{k}, & \text { when POI is absent } \\ \rho_{0} D^{-\beta}+w_{k}, & \text { when POI is present }\end{cases}
$$

where $k=1,2, \ldots ; \rho_{0}$ is the signal strength measured at $1 \mathrm{~m}$ from the POI location; $D$ is the distance between the POI and the sensor, as shown in Fig. 1; and $w_{k}$ is the AWGN corrupting the observation. Let us consider binary quantization, where the sensor will flip the bit received from the interrogator if and only if $\rho_{k}$ is larger than a sensing threshold $\rho^{*}$. As a result, the error probability for $C_{\mathrm{NI}}$ in Fig. 2(b) is given by

$$
q_{\mathrm{NI}}=\operatorname{Pr}\left\{w_{k}>\rho^{*} \mid C_{\mathrm{NI}}\right\}=\mathcal{Q}\left(\frac{\rho^{*}}{\sigma_{w}}\right)
$$

where $\sigma_{w}$ is the standard deviation of the noise. On the other hand, the error probability for $C_{\mathrm{I}}$ in Fig. 2(b) is

$q_{\mathrm{I}}=\operatorname{Pr}\left\{\rho_{0} D^{-\beta}+w_{k} \leq \rho^{*} \mid C_{\mathrm{I}}\right\}=1-\mathcal{Q}\left(\frac{\rho^{*}-\rho_{0} D^{-\beta}}{\sigma_{w}}\right)$.

Note that $q_{\mathrm{NI}}=q_{\mathrm{I}}$ if and only if $\rho^{*}=\rho_{0} D^{-\beta} / 2$. In this case, the DMC in Fig. 2(b) would reduce to a standard BSC.

\section{B. Time-Varying Wireless Channel and Time-Invariant POI}

When the wireless channel is time varying and the POI is time invariant, the information transfer over a CISN can be described through the layered FSMC-DMC model presented in Section II-B2.

1) AMF System: We will first consider an AMF scheme. If the nonstationarity of the propagation environment is caused by the motion of sensors (e.g., sensing devices mounted on moving vehicles), narrowband fading will occur, which is described as [30]

$$
y_{\mathbf{R}}(t)=h_{\mathrm{F}} h_{\mathrm{B}} \tilde{h}_{\mathrm{F}}(t) \tilde{h}_{\mathrm{B}}(t) x_{\mathbf{S}}(t)+h_{\mathrm{F}} \tilde{h}_{\mathrm{F}}(t) n(t)+n(t)
$$

where $h_{\mathrm{B}}$ and $h_{\mathrm{F}}$ are given in (10). $\tilde{h}_{\mathrm{B}}(t)$ and $\tilde{h}_{\mathrm{F}}(t)$ are the unit-variance complex fading coefficients for the backward and forward channels, respectively. In general, it is difficult to obtain a closed-form statistical analysis of the instantaneous SNR for (21) [30]. Nevertheless, the problem can be simplified when $\left|h_{\mathrm{F}} \tilde{h}_{\mathrm{F}}(t)\right|^{2} \ll 1$, which is satisfied if $\mathcal{P}_{\mathbf{S}}^{\mathrm{t}} \ll \mathcal{P}_{\mathbf{T}}^{\mathrm{t}}$ following (10) and (11). This condition is common because the sensors are usually power constrained, whereas the interrogator, like the RFID reader, is of high transmit power [11]-[19]. In this case, 
the noise term $h_{\mathrm{F}} \tilde{h}_{\mathrm{F}}(t) n(t)$ would be much less than $n(t)$ and, hence, can be neglected.

If the fading envelopes for both up and down links exhibit an identical and independent Rayleigh distribution, the overall envelope $\alpha=\left|\tilde{h}_{\mathrm{B}}\right|\left|\tilde{h}_{\mathrm{F}}\right|$ is double-Rayleigh faded [30], i.e.,

$$
f_{\alpha}(\alpha)=4 \alpha K_{0}(2 \alpha) .
$$

The $K_{0}(\cdot)$ function is the zeroth-order Bessel function of the second kind. We assume isotropic antennas operating in a 2-D isotropic scattering environment [30]. The level crossing rate (LCR) of $\alpha$ at level $\alpha^{*}$, which is defined as the rate at which the channel envelope crosses $\alpha^{*}$, is [30]

$$
\begin{aligned}
L_{\alpha}\left(\alpha^{*}\right)=\frac{4 \sqrt{\pi} \alpha^{*}}{\sqrt{2}} \int_{0}^{\infty} \frac{1}{z^{2}} \exp [ & \left.-\frac{\left(\alpha^{*}\right)^{2}+z^{4}}{z^{2}}\right] \\
& \times \sqrt{\nu^{2} z^{4}+\nu^{2}\left(\alpha^{*}\right)^{2}} d z
\end{aligned}
$$

where $\nu$ is the maximum Doppler shift induced by the motion of the sensor.

Next, we construct the simplest two-state FSMC [or the Gilbert-Elliott channel (GEC)], as illustrated in Fig. 2(c), where each state corresponds to a specific channel quality classified according to a threshold level $\alpha^{*}$ [27]. That is, the double-Rayleigh fading channel is said to be in state $S_{0}$ (or the "bad" state in the GEC) and state $S_{1}$ (or the "good" state in the GEC) if the fading envelope is in the intervals $\left[0, \alpha^{*}\right)$ and $\left[\alpha^{*}, \infty\right]$, respectively. Following the methodology in [27], the steady-state probability of each state is

$$
\begin{aligned}
& \Pi_{0}=\int_{0}^{\alpha^{*}} f_{\alpha}(\alpha) d \alpha=\int_{0}^{\alpha^{*}} 4 \alpha K_{0}(2 \alpha) d \alpha \\
& \Pi_{1}=1-\Pi_{0}=1-\int_{0}^{\alpha^{*}} 4 \alpha K_{0}(2 \alpha) d \alpha .
\end{aligned}
$$

The state transition probabilities are related to the LCRs and the steady-state probabilities through the following relationships [27]:

$$
\begin{aligned}
& P_{0 \rightarrow 1}=\frac{L_{\alpha}\left(\alpha^{*}\right) T_{s}}{\Pi_{0}} \\
& P_{1 \rightarrow 0}=\frac{L_{\alpha}\left(\alpha^{*}\right) T_{s}}{\Pi_{1}}
\end{aligned}
$$

where $T_{s}$ is the symbol period. Other state transition probabilities are given as $P_{0 \rightarrow 0}=1-P_{0 \rightarrow 1}$ and $P_{1 \rightarrow 1}=1-P_{1 \rightarrow 0}$. Finally, the average crossover probability for each state is [27]

$$
\begin{aligned}
p_{0} & =\frac{\int_{0}^{\alpha^{*}} p(\alpha) f_{\alpha}(\alpha) d \alpha}{\Pi_{0}} \\
= & \frac{\int_{0}^{\alpha^{*}} \mathcal{Q}\left(\sqrt{\frac{2 \mathcal{P}_{\mathbf{S}}^{\mathrm{t}} A^{2} \alpha^{2} d_{2}^{-\kappa} \gamma}{1+\sigma_{n}^{2} /\left(\mathcal{P}_{\mathbf{T}}^{\mathrm{t}} A^{2} d_{1}^{-\kappa}\right)}}\right) 4 \alpha K_{0}(2 \alpha) d \alpha}{\int_{0}^{\alpha^{*}} 4 \alpha K_{0}(2 \alpha) d \alpha}
\end{aligned}
$$

$$
\begin{aligned}
p_{1} & =\frac{\int_{\alpha^{*}}^{\infty} p(\alpha) f_{\alpha}(\alpha) d \alpha}{\Pi_{1}} \\
& =\frac{\int_{\alpha^{*}}^{\infty} \mathcal{Q}\left(\sqrt{\frac{2 \mathcal{P}_{\mathbf{S}}^{\mathrm{t}} A^{2} \alpha^{2} d_{2}^{-\kappa} \gamma}{1+\sigma_{n}^{2} /\left(\mathcal{P}_{\mathbf{T}}^{\mathrm{t}} A^{2} d_{1}^{-\kappa}\right)}}\right) 4 \alpha K_{0}(2 \alpha) d \alpha}{1-\int_{0}^{\alpha^{*}} 4 \alpha K_{0}(2 \alpha) d \alpha}
\end{aligned}
$$

where $p(\alpha)$ is the crossover probability for a specific fading coefficient $\alpha$ and is derived following (13) and assuming $\mathcal{P}_{\mathbf{S}}^{\mathrm{t}} \ll$ $\mathcal{P}_{\mathrm{T}}^{\mathrm{t}}$. The foregoing analysis can easily be extended to more general FSMCs with more than two states.

2) DMF System: When a DMF scheme is adopted, the fading signal received at the sensor is

$$
y_{\mathbf{S}}(t)=h_{\mathrm{B}} \tilde{h}_{\mathrm{B}}(t) x_{\mathbf{T}}(t)+n(t) .
$$

Following Section III-B1, the fading envelope $\alpha_{\mathrm{B}}=\left|\tilde{h}_{\mathrm{B}}\right|$ is Rayleigh distributed with unit variance per complex dimension given as

$$
f_{\alpha_{\mathrm{B}}}\left(\alpha_{\mathrm{B}}\right)=2 \alpha_{\mathrm{B}} \exp \left(-\alpha_{\mathrm{B}}^{2}\right) .
$$

The LCR at an amplitude boundary threshold $\alpha_{\mathrm{B}}^{*}$ is [42]

$$
L_{\alpha_{\mathrm{B}}}\left(\alpha_{\mathrm{B}}^{*}\right)=\sqrt{2 \pi} \alpha_{\mathrm{B}}^{*} \nu \exp \left[-\left(\alpha_{\mathrm{B}}^{*}\right)^{2}\right]
$$

with $\nu$ being the maximum Doppler frequency shift.

For this backward connection, we can easily establish the GEC, as shown in Fig. 4(a), following the practice in [27]. The channel is said to be in state $S_{\mathrm{B}, 0}$ and state $S_{\mathrm{B}, 1}$ if the fading envelope is in the intervals $\left[0, \alpha_{\mathrm{B}}^{*}\right)$ and $\left[\alpha_{\mathrm{B}}^{*}, \infty\right]$, respectively. The steady-state probabilities are obtained as

$$
\begin{aligned}
& \Pi_{\mathrm{B}, 0}=\int_{0}^{\alpha_{\mathrm{B}}^{*}} f_{\alpha_{\mathrm{B}}}\left(\alpha_{\mathrm{B}}\right) d \alpha_{\mathrm{B}}=1-\exp \left[-\left(\alpha_{\mathrm{B}}^{*}\right)^{2}\right] \\
& \Pi_{\mathrm{B}, 1}=1-\Pi_{\mathrm{B}, 0}=\exp \left[-\left(\alpha_{\mathrm{B}}^{*}\right)^{2}\right] .
\end{aligned}
$$

Finally, the state transition probabilities $P_{\mathrm{B}, i \rightarrow j}(i, j \in\{0,1\})$ and the average crossover probabilities $p_{\mathrm{B}, i}(i \in\{0,1\})$ can be calculated following (26)-(29) by substituting the relevant parameters for the backward channel. In a similar manner, we can construct the GEC for the sensor-to-sink connection as depicted in Fig. 4(b), where the subscript $F$ is used to replace the subscript ${ }_{\mathrm{B}}$ to indicate the forward channel.

The cascading of two GECs is equivalent to a four-state FSMC, as illustrated in Fig. 4(c), where each state is defined as $S_{i j} \triangleq\left\{S_{\mathrm{B}}=S_{\mathrm{B}, i}, S_{\mathrm{F}}=S_{\mathrm{F}, j}\right\} \quad(i, j \in\{0,1\})$. Assuming independence between the backward and forward channels, the steady-state probabilities are $\Pi_{i j}=\Pi_{\mathrm{B}, i} \times$ $\Pi_{\mathrm{F}, j}$, and the state transition probabilities are $P_{i_{1} j_{1} \rightarrow i_{2} j_{2}}=$ $P_{\mathrm{B}, i_{1} \rightarrow i_{2}} \times P_{\mathrm{F}, j_{1} \rightarrow j_{2}}\left(i_{1}, j_{1}, i_{2}, j_{2} \in\{0,1\}\right)$. By applying (4), the crossover probabilities are calculated to be $p_{i j}=p_{\mathrm{B}, i}+$ $p_{\mathrm{F}, j}-2 p_{\mathrm{B}, i} p_{\mathrm{F}, j}$.

3) POI: For a time-invariant POI, the formulations in (18)-(20) can readily be applied to determine its DMC model parameters, which govern the transition between $C_{\mathrm{NI}}$ and $C_{\mathrm{I}}$ [cf. Fig. 2(b)]. 


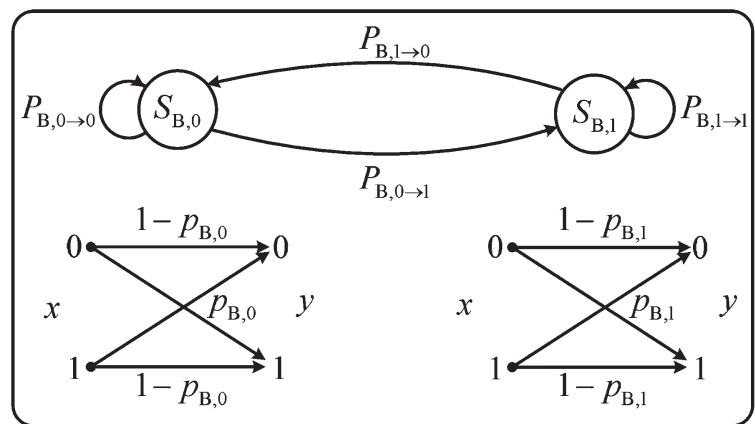

(a)

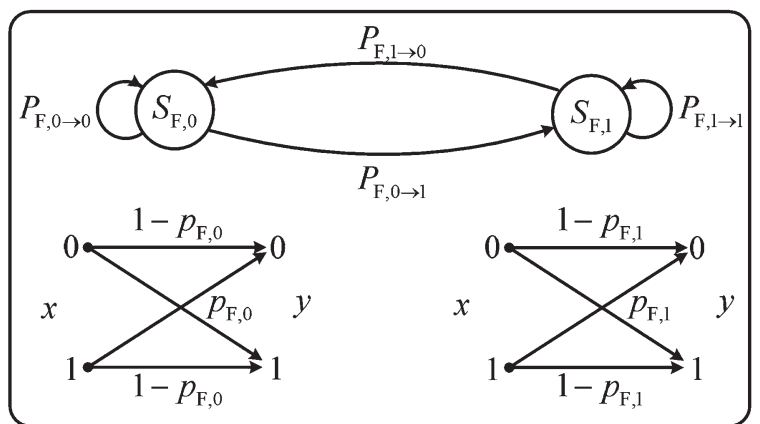

(b)

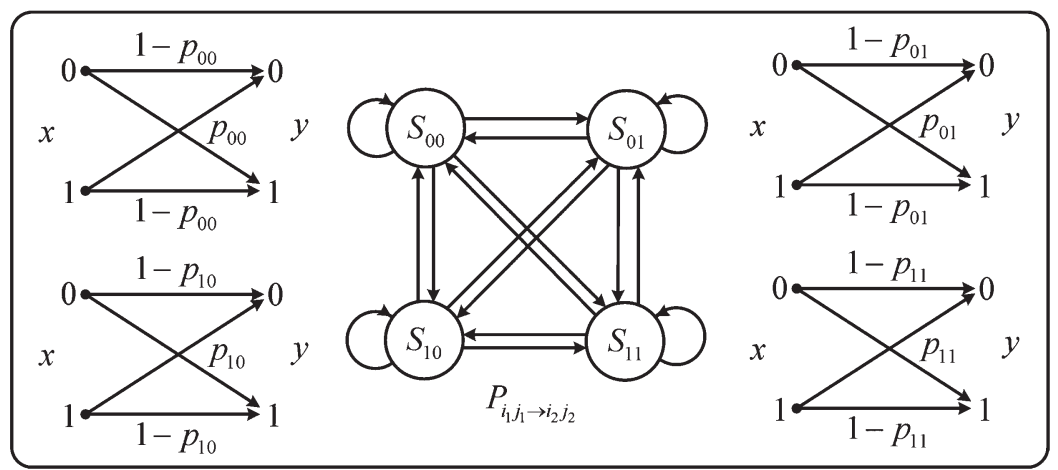

(c)

Fig. 4. (a) FSMC for the backward link. (b) FSMC for the forward link. (c) Equivalent FSMC for the noninverting DMF system. The corresponding inverting FSMC can easily be constructed following Fig. 2(c).

\section{Time-Invariant Wireless Channel and Time-Varying POI}

When the propagation channel is time invariant and the POI is time varying, the information transfer over a CISN can be modeled using the layered DMC-FSMC model discussed in Section II-B2.

1) $A M F$ and $D M F$ : For a time-invariant communication link, its BSCs can be established using (9)-(17) [cf. Fig. 2(a)].

2) POI: Let us consider an isotropic time-varying POI source. The received signal strength at the sensor $\rho_{k}$, after appropriate sampling and processing, is given by

$$
\rho_{k}= \begin{cases}w_{k}, & \text { when POI is absent } \\ \hat{\rho}_{k}+w_{k}, & \text { when POI is present }\end{cases}
$$

where $\hat{\rho}_{k}$ is the sampled version of a continuous-time nonnegative random process $\hat{\rho}(t)$. For illustration purposes, $\hat{\rho}(t)$ is assumed to follow a lognormal distribution. In other words, $\zeta=10 \log _{10} \hat{\rho}$ is normally distributed with mean $\zeta_{\mathrm{m}}$ and standard deviation $\sigma_{\zeta}$, as given by

$$
f_{\zeta}(\zeta)=\frac{1}{\sigma_{\zeta} \sqrt{2 \pi}} \exp \left[-\frac{\left(\zeta-\zeta_{\mathrm{m}}\right)^{2}}{2 \sigma_{\zeta}^{2}}\right] .
$$

Here, $\zeta_{\mathrm{m}}=10 \log _{10}\left(\rho_{0} D^{-\beta}\right)$ is the mean received signal strength at the sensor (in decibels) in the presence of POI, as given in (18). The LCR of $\zeta$ at level $\zeta^{*}$ is [44]

$$
L_{\zeta}\left(\zeta^{*}\right)=\frac{\sqrt{\varrho}}{2 \pi} \exp \left[-\frac{\left(\zeta^{*}-\zeta_{\mathrm{m}}\right)^{2}}{2 \sigma_{\zeta}^{2}}\right]
$$

where $\varrho$ is related to the autocorrelation function $R_{\zeta \zeta}(\Delta t)$ of the underlying Gaussian process $\zeta(t)$ as

$$
\varrho=-\left.\frac{d^{2}}{d \Delta t^{2}} R_{\zeta \zeta}(\Delta t)\right|_{\Delta t=0}=-\ddot{R}_{\zeta \zeta}(0) .
$$


If the temporal correlation properties of $\zeta(t)$ follow a Gaussian model [44]

$$
R_{\zeta \zeta}(\Delta t)=\exp \left[-\left(\frac{\Delta t}{\tau_{\mathrm{D}}}\right)^{2}\right]
$$

with $\tau_{\mathrm{D}}$ being the decorrelation time, it follows that the quantity $\varrho=-\ddot{R}_{\zeta \zeta}(0)=2 / \tau_{\mathrm{D}}^{2}$, and $L_{\zeta}\left(\zeta^{*}\right)$ can easily be analyzed using (37).

The next step is to construct a two-state FSMC model to characterize the transition between $C_{\mathrm{NI}}$ and $C_{\mathrm{I}}$, as illustrated in Fig. 2(d). By applying the same approach used to define an FSMC in fading channels, as discussed in Section III-B, the sensing link is said to be in state $\mathbb{S}_{0}$ and state $\mathbb{S}_{1}$ if the received POI strength $\zeta$ is in the intervals $\left(-\infty, \zeta^{*}\right)$ and $\left[\zeta^{*}, \infty\right)$, respectively. The steady-state probability of each state is derived by integrating the distribution function of the received POI over the corresponding region, where

$$
\begin{aligned}
& \pi_{0}=\int_{-\infty}^{\zeta^{*}} f_{\zeta}(\zeta) d \zeta=1-\mathcal{Q}\left(\frac{\zeta^{*}-\zeta_{\mathrm{m}}}{\sigma_{\zeta}}\right) \\
& \pi_{1}=1-\pi_{0}=\mathcal{Q}\left(\frac{\zeta^{*}-\zeta_{\mathrm{m}}}{\sigma_{\zeta}}\right) .
\end{aligned}
$$

The state transition probabilities can be approximated as

$$
\begin{gathered}
Q_{0 \rightarrow 1}=\frac{L_{\zeta}\left(\zeta^{*}\right) \tau_{s}}{\pi_{0}} \\
Q_{1 \rightarrow 0}=\frac{L_{\zeta}\left(\zeta^{*}\right) \tau_{s}}{\pi_{1}}
\end{gathered}
$$

with $\tau_{s}$ being the sampling period. Other state transition probabilities are given as $Q_{0 \rightarrow 0}=1-Q_{0 \rightarrow 1}$ and $Q_{1 \rightarrow 1}=1-$ $Q_{1 \rightarrow 0}$. Finally, the average error probabilities for $C_{\mathrm{NI}}$ and $C_{\mathrm{I}}$ in each state are

$$
\begin{aligned}
q_{\mathrm{NI}, 0} & =\frac{\int_{-\infty}^{\zeta^{*}} q_{\mathrm{NI}}(\zeta) f_{\zeta}(\zeta) d \zeta}{\pi_{0}}=\mathcal{Q}\left(\frac{\rho^{*}}{\sigma_{w}}\right) \\
q_{\mathrm{I}, 0} & =\frac{\int_{-\infty}^{\zeta^{*}} q_{\mathrm{I}}(\zeta) f_{\zeta}(\zeta) d \zeta}{\pi_{0}} \\
& =\frac{\int_{-\infty}^{\zeta^{*}}\left[1-\mathcal{Q}\left(\frac{\rho^{*}-10 \frac{\zeta}{10}}{\sigma_{w}}\right)\right] \frac{1}{\sigma_{\zeta} \sqrt{2 \pi}} \exp \left[-\frac{\left(\zeta-\zeta_{\mathrm{m}}\right)^{2}}{2 \sigma_{\zeta}^{2}}\right] d \zeta}{1-\mathcal{Q}\left(\frac{\zeta^{*}-\zeta_{\mathrm{m}}}{\sigma_{\zeta}}\right)}
\end{aligned}
$$

$$
\begin{aligned}
q_{\mathrm{NI}, 1} & =\frac{\int_{\zeta^{*}}^{\infty} q_{\mathrm{NI}}(\zeta) f_{\zeta}(\zeta) d \zeta}{\pi_{1}}=\mathcal{Q}\left(\frac{\rho^{*}}{\sigma_{w}}\right) \\
q_{\mathrm{I}, 1} & =\frac{\int_{\zeta^{*}}^{\infty} q_{\mathrm{I}}(\zeta) f_{\zeta}(\zeta) d \zeta}{\pi_{1}} \\
& =\frac{\int_{\zeta^{*}}^{\infty}\left[1-\mathcal{Q}\left(\frac{\rho^{*}-10 \frac{\zeta}{10}}{\sigma_{w}}\right)\right] \frac{1}{\sigma_{\zeta} \sqrt{2 \pi}} \exp \left[-\frac{\left(\zeta-\zeta_{\mathrm{m}}\right)^{2}}{2 \sigma_{\zeta}^{2}}\right] d \zeta}{\mathcal{Q}\left(\frac{\zeta^{*}-\zeta_{\mathrm{m}}}{\sigma_{\zeta}}\right)} .
\end{aligned}
$$

Here, $q_{\mathrm{NI}}(\zeta)$ and $q_{\mathrm{I}}(\zeta)$ are the error probabilities for $C_{\mathrm{NI}}$ and $C_{\mathrm{I}}$, respectively, conditioned on a specific $\zeta$. They are derived following (19) and (20). Note that both $q_{\mathrm{NI}, 0}$ and $q_{\mathrm{NI}, 1}$ are fixed and independent of $\zeta$. The foregoing analysis can readily be extended to more general FSMC models with more than two states.

\section{Time-Varying Wireless Channel and POI}

When both the wireless channel and the POI are time varying, the layered FSMC model in Section II-B3 is appropriate for defining the input-output relationship in a CISN. The formulations in (21)-(47) can be employed to determine the model parameters in Fig. 2(c) and (d), respectively.

\section{NumericAl ExAMPLES}

We consider a time-invariant communication channel and a time-varying sensing link in the following numerical examples. The interrogator and the sink are located at $\mathbf{T}(100 \mathrm{~m}, 0 \mathrm{~m})$ and $\mathbf{R}(0 \mathrm{~m}, 0 \mathrm{~m})$, respectively, in the Cartesian coordinate shown in Fig. 3. A total number of 100 sensors are uniformly distributed within a square defined by $\{10 \mathrm{~m} \leq \mathcal{X} \leq 90 \mathrm{~m}\} \cap$ $\{10 \mathrm{~m} \leq \mathcal{Y} \leq 90 \mathrm{~m}\}$, and the POI is located at $(50 \mathrm{~m}, 50 \mathrm{~m})$. For the communication channel, the reference SNRs at the interrogator and the sensor are $\mathcal{P}_{\mathbf{T}}^{\mathrm{t}} A^{2} \gamma=\mathcal{P}_{\mathbf{T}}^{\mathrm{t}} A^{2} / \sigma_{n}^{2}=30 \mathrm{~dB}$ and $\mathcal{P}_{\mathbf{S}}^{\mathrm{t}} A^{2} \gamma=\mathcal{P}_{\mathbf{S}}^{\mathrm{t}} A^{2} / \sigma_{n}^{2}=20 \mathrm{~dB}$, respectively [see also (13), (15), and (17)]. It is further assumed that the path loss exponent is $\kappa=2$. For the sensing link, the sampling period normalized with respect to the decorrelation time is $\tau_{s} / \tau_{\mathrm{D}}=0.2$. The standard deviation of the AWGN is $\sigma_{w}=1 \mathrm{u}$, where "u" denotes the measurement unit of the POI. The received POI strength at $1 \mathrm{~m}$ is $\rho_{0}=50 \mathrm{u}$, the decision threshold is set to be $\rho^{*}=2 \mathrm{u}$, and the path loss factor is $\beta=1$. Finally, the standard deviation of the lognormally distributed time-varying process $\zeta(t)$ is given by $\sigma_{\zeta}=3 \mathrm{dBu}$, and the threshold for the state transition to occur is $\zeta^{*}=3 \mathrm{dBu}$.

The numerical results for the communication link are illustrated in Fig. 5. Fig. 5(a) and (b) shows the contours of crossover probabilities for AMF and DMF systems, respectively. Comparing Fig. 5(a) with 5(b) shows that DMF achieves slightly better error performance (smaller crossover probabilities) as compared with AMF. Furthermore, all the contours are asymmetric with reference to the orientation of the interrogator and the sink, owing to the unbalanced influence of the backward and forward links on the overall error performance. In general, sensors closer to the sink lead to lower crossover probabilities. Fig. 5(c) demonstrates four cross-sectional views of the doubledirectional CCAS with AMF at four different ranges of AOD $\Omega$ : $\left[10^{\circ}, 20^{\circ}\right],\left[30^{\circ}, 40^{\circ}\right],\left[50^{\circ}, 60^{\circ}\right]$, and $\left[70^{\circ}, 80^{\circ}\right]$. It is evident that close-in sensors with smaller values of AOD $\Omega$ and AOA $\Psi$ result in larger channel capacities. This is due to the reduced propagation loss, as can be predicted from (13), (15), and (17). Fig. 5(d) illustrates the cross-sectional views of the doubledirectional CCAS with DMF. Similar trends to those in Fig. 5(c) can be observed. Finally, larger channel capacities are obtained in Fig. 5(d), as compared with Fig. 5(c), demonstrating the potential advantage of DMF over AMF in this particular case. 


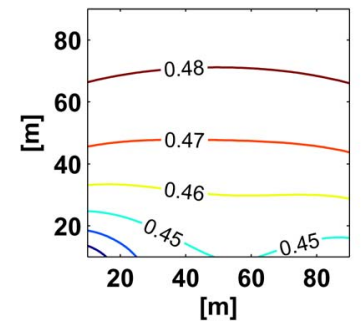

(a)

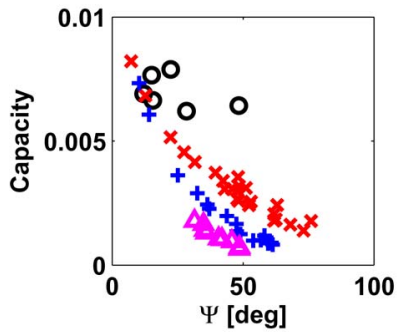

(c)

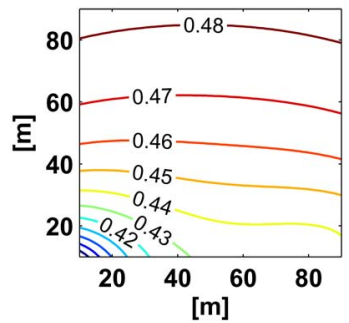

(b)

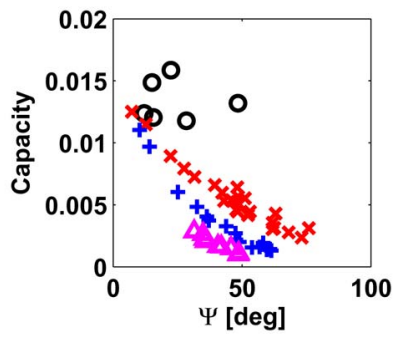

(d)
Fig. 5. Contours of crossover probabilities for the time-invariant communication link. (a) AMF and (b) DMF; cross-sectional views of the doubledirectional CCAS with (c) AMF and (d) DMF. The legend for (c) and (d) is as follows: $\circ: \Omega \in\left[10^{\circ}, 20^{\circ}\right], \times: \Omega \in\left[30^{\circ}, 40^{\circ}\right],+: \Omega \in\left[50^{\circ}, 60^{\circ}\right]$, $\triangle: \Omega \in\left[70^{\circ}, 80^{\circ}\right]$.

This also agrees well with the crossover probability data in Fig. 5(a) and (b).

The results for the time-varying sensing link are plotted in Figs. 6 and 7. Fig. 6 shows that, as a sensor locates farther away from the POI (hence, the average received POI strength decreases), the probability that the sensing process stays in the "bad" state $\mathbb{S}_{0}$, i.e., $\pi_{0}$, increases. In contrast, the probability that it stays in the "good" state $\mathbb{S}_{1}$, i.e., $\pi_{1}$, decreases. Furthermore, a larger distance between the POI and the sensor leads to a smaller state transition probability from $\mathbb{S}_{0}$ to $\mathbb{S}_{1}$, i.e., $Q_{0 \rightarrow 1}$. An opposite trend can be found for $Q_{1 \rightarrow 0}$.

Fig. 7(a) and (b) shows that the average error probabilities for $C_{\mathrm{I}}$ increase with the distance between the POI and the sensor for both $\mathbb{S}_{0}$ and $\mathbb{S}_{1}$. On average, a much larger error probability is obtained in the former case. Note that the error probability for $C_{\mathrm{NI}}$ is equal to $\mathcal{Q}(2)$ [see also (44) and (46)], which is independent of the channel states and sensor locations. Fig. 7(c) depicts four cross sections of the double-directional CIAS in the "bad" state $\mathbb{S}_{0}$ at four different ranges of $\Omega$. A larger Bhattacharyya distance is observed when the sensor is closer to the POI. More specifically, larger error exponents resulted when $\Omega$ is in the interval of $\left[30^{\circ}, 40^{\circ}\right]$ or $\left[50^{\circ}, 60^{\circ}\right.$, and $\Psi$ is around $45^{\circ}$. Fig. $7(d)$ plots the cross sections of the double-directional CIAS in the "good" state $\mathbb{S}_{1}$. Similar trends to those in Fig. 7(c) can be found, except that much larger Bhattacharyya distances are obtained in this case. Finally, all the contours are symmetric with reference to the POI location, as shown in Figs. 6 and 7(a) and (b), owing to the omnidirectional characteristics of the POI source.

To study the influence of sensor distribution on system performance, we further consider the scenario that the sensor density gradually tapers off with distance from the POI. Specifically, we look into the model where the sensor density around the POI follows a truncated Gaussian distribution $f_{D}(D)=$

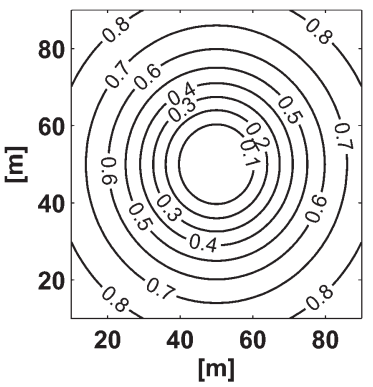

(a)

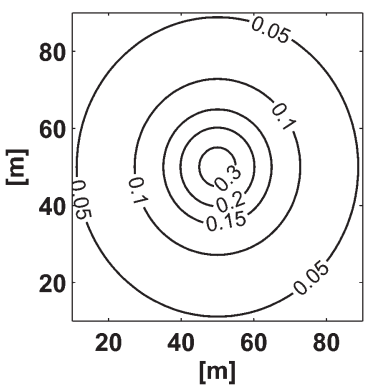

(c)

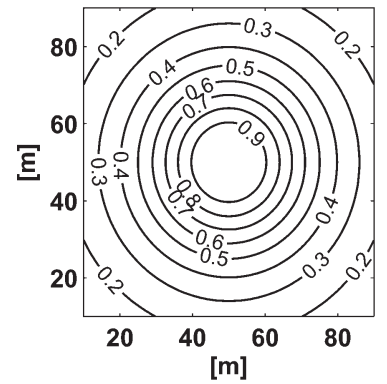

(b)

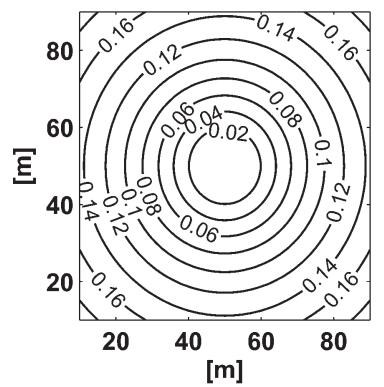

(d)
Fig. 6. Contours of (a) $\pi_{0}$, (b) $\pi_{1}$, (c) $Q_{0 \rightarrow 1}$, and (d) $Q_{1 \rightarrow 0}$ for the timevarying sensing link.

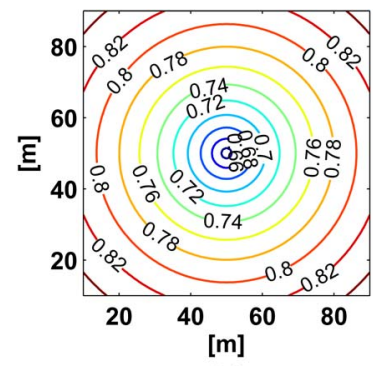

(a)

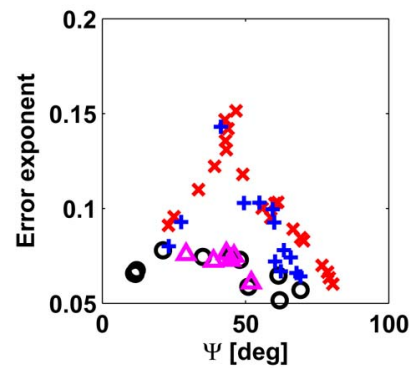

(c)

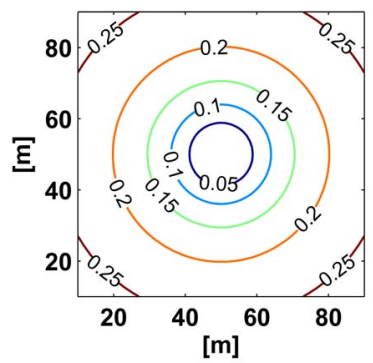

(b)

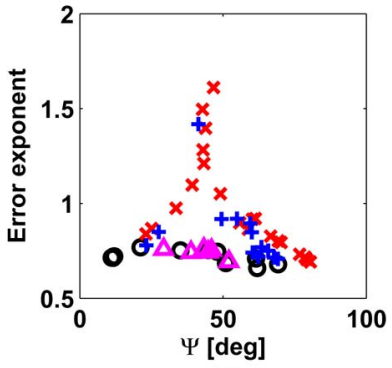

(d)
Fig. 7. Contours of the average error probabilities for $C_{\mathrm{I}}$ in (a) state $\mathbb{S}_{0}$ and (b) state $\mathbb{S}_{1}$ for the time-varying sensing link; cross-sectional views of the double-directional CIAS for (c) state $\mathbb{S}_{0}$ and $(d)$ state $\mathbb{S}_{1}$. The legend for (c) and (d) is as follows: $\circ: \Omega \in\left[10^{\circ}, 20^{\circ}\right], \times: \Omega \in\left[30^{\circ}, 40^{\circ}\right],+: \Omega \in$ $\left[50^{\circ}, 60^{\circ}\right], \triangle: \Omega \in\left[70^{\circ}, 80^{\circ}\right]$.

$\varsigma \exp \left(-D^{2} / 2 \sigma_{D}^{2}\right)$, where $D$ is the distance between the sensor and the POI, and $\varsigma$ is a normalization factor to ensure that $f_{D}(D)$ is a valid probability density function after truncation of sensor distribution beyond the network boundary. The standard deviation $\sigma_{D}$ is a measure of the effective width of the sensor region. This model is analogous to the Gaussian scatter density model proposed in the context of geometric channel modeling for multipath propagation [45]. We further assume that a total 


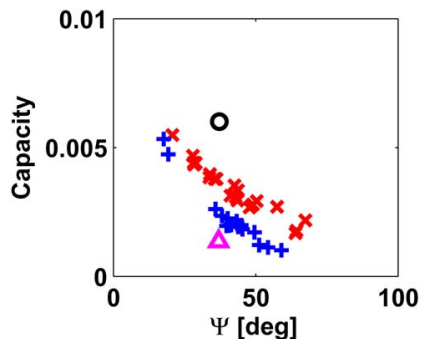

(a)

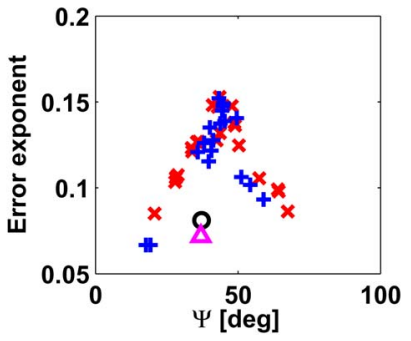

(c)

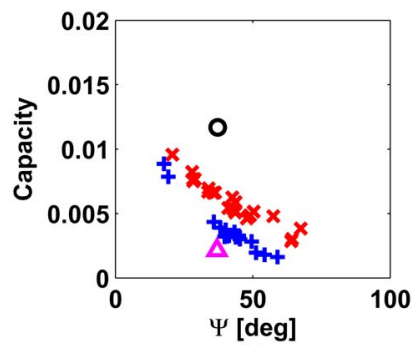

(b)

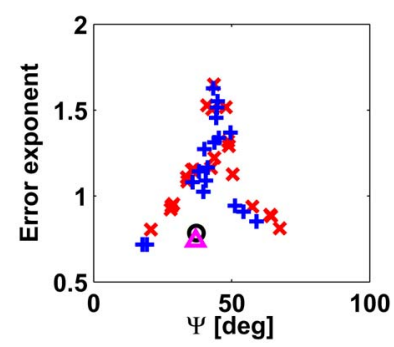

(d)
Fig. 8. Cross-sectional views of the double-directional CCAS for Gaussian sensor density network. (a) AMF and (b) DMF; cross-sectional views of the double-directional CIAS for Gaussian sensor density network. (c) State $\mathbb{S}_{0}$ and (d) state $\mathbb{S}_{1}$. The legend for all the subplots is as follows: $\circ: \Omega \in\left[10^{\circ}, 20^{\circ}\right]$, $\times: \Omega \in\left[30^{\circ}, 40^{\circ}\right],+: \Omega \in\left[50^{\circ}, 60^{\circ}\right], \triangle: \Omega \in\left[70^{\circ}, 80^{\circ}\right]$.

number of 100 sensors are randomly scattered in the same square region as in the previous examples, and the sensor placement follows the Gaussian density model with $\sigma_{D}=$ $20 \mathrm{~m}$. Fig. 8 illustrates the corresponding cross-sectional views of the double-directional CCAS and CIAS at four different intervals of $\Omega$. Comparing Fig. 8(a) and (b) with Fig. 5(c) and (d) indicates that the Gaussian sensor density network reduces the number of nodes with high channel capacities. On the other hand, it leads to increased number of nodes with large Bhattacharyya distances, as can be observed by comparing Fig. 8(c) and (d) with Fig. 7(c) and (d). Apparently, there is a tradeoff between the communication and sensing link qualities when a tapered sensor distribution is employed to replace the uniform pattern. It is worth emphasizing that the proposed analytical framework can also be applied for any other sensor density models. As a result, optimal distributions could be identified based on various predefined design criteria.

Essentially, Figs. 5, 7, and 8 provide intuitive and quantitative answers to the following two important questions. First, how should the sensor relays be distributed to achieve an optimal error performance of the communication and sensing links, and second, what would be the suitable beam-scanning ranges at the interrogator and the sink to obtain the maximum communication channel capacity and the best achievable error exponent for the sensing link?

\section{Applications of the Proposed Models}

In this section, we briefly discuss some important applications of the proposed models for analysis of CISNs.

1) Cognitive Sensing: Knowledge-based adaptive transmission is of practical significance in a CISN for the effective monitoring and transmission of POI data. In the canonical problem posed in Section II-D, the interrogator intelligently and adaptively customizes its transceiver operation (i.e., $\mathcal{B}_{\mathbf{T}}^{\hat{k}+1}$, $\mathcal{B}_{\mathbf{R}}^{\hat{k}+1}, X^{\hat{k}+1}, \mathcal{C}^{\hat{k}+1}$ ) based on some side information about the environment and the system (i.e., $\mathcal{B}_{\mathbf{T}}^{\hat{k}}, \mathcal{B}_{\mathbf{R}}^{\hat{k}}, X^{\hat{k}}, Y^{\hat{k}}, \mathcal{C}^{\hat{k}}, \mathbf{L}^{\hat{k}}$, $\mathbf{P}^{\hat{k}}$ ) [1], [5]. One key advantage of using the layered DMC and FSMC models for cognitive sensing is their mathematical tractability. For example, in FSMC, there is only a finite number of states that need to be estimated at the receiver. This favorably compares with continuous-valued channels with an infinitestate space and is particularly useful in the low SNR regime, where the reliability of channel estimation is more important than precision [26].

2) Network Performance Assessment: The layered DMC and FSMC models are well suited to represent sensing errors that may be observed in noisy or time-varying environments. Mathematically tractable expressions for system performance measures may be obtained, which include bit error rate, data throughput, fade duration distribution, channel capacity, and capacity-achieving input distribution.

It is worth emphasizing that all these measures are related to the sensing process, as mentioned in Section II-D, which is essentially the differential information between the inputs and outputs of the communication link, rather than the input/output data themselves. This is in contrast to a traditional wireless communication system, in which the object of interest is the input/output information of the channel.

3) Network Simulation: The layered DMC and FSMC models are useful in providing faster and more convenient CISN simulations. Once the DMC error probabilities, FSMC states, state transition probabilities, and crossover probability in each state are determined, channel simulation comprises generating several sequences of independent random variables. Each sequence either decides whether a state transition has taken place or whether an error has occurred [26]. The procedures are much simpler than the generation of continuous-valued random processes.

A fundamental challenge, however, is to design the appropriate layered DMC and FSMC models that provide accurate and reliable system performance evaluation. Important issues, including the number of states and the Markov memory order, should be investigated based on various criteria for accuracy.

\section{CONCLUSION}

We have presented a comprehensive study of CISN channel modeling. The layered DMC and FSMC models have been proposed to characterize information transfer over CISNs, where the communication link is underlaid by the sensing link. These two processes are separately characterized using either DMC or FSMC, depending on the stationarity of the underlying stochastic processes. We have also proposed two sensor encoding strategies in CISNs (including AMF and DMF) and introduced the concept of double-directional CCAS and CIAS to facilitate the network-level assessment of link reliability. The models provided in this paper provide critical insight into both theoretical and experimental analyses of CISNs.

Further research efforts are required to provide the laboratory experimental implementation of CISNs. As future works, the 
advantages and limitations of the proposed cognitive sensing framework need to be verified by real-life prototypes and experimentation. Various designs of RFID sensor tags available in the existing literature (e.g., multiport tag antennas used as sensors [12], passive chipless RFID sensor system [17], wireless identification and sensing platform [11]) can be applied as sensors in WSNs. Moreover, the integration of a long-range RFID reader [11] and a CR by incorporating an internal feedback loop poses a great design challenge. Eventually, such an integrated system can be customized for the realization and empirical testing of CISNs.

\section{REFERENCES}

[1] S. Haykin, "Cognitive radar: A way of the future," IEEE Signal Process. Mag., vol. 23, no. 1, pp. 30-40, Jan. 2006.

[2] N. A. Goodman, P. R. Venkata, and M. A. Neifeld, "Adaptive waveform design and sequential hypothesis testing for target recognition with active sensors," IEEE J. Sel. Topics Signal Process., vol. 1, no. 1, pp. 105-113, Jun. 2007.

[3] U. Gunturkun, "Toward the development of radar scene analyzer for cognitive radar," IEEE J. Ocean. Eng., vol. 35, no. 2, pp. 303-313, Apr. 2010.

[4] R. A. Romero and N. A. Goodman, "Waveform design in signaldependent interference and application to target recognition with multiple transmissions," IET Radar Sonar Navig., vol. 3, no. 4, pp. 328-340, Aug. 2009.

[5] Y. Chen and P. Rapajic, "Ultra-wideband cognitive interrogator network: Adaptive illumination with active sensors for target localisation," IET Commun., vol. 4, no. 5, pp. 573-584, Mar. 2010.

[6] D. Culler, D. Estrin, and M. Srivastava, "Overview of sensor networks," Computer, vol. 37, no. 8, pp. 41-49, Aug. 2004.

[7] B. Krishnamachari, Networking Wireless Sensors. Cambridge, U.K.: Cambridge Univ. Press, 2006.

[8] R. Madan, S. Cui, S. Lall, and A. Goldsmith, "Cross-layer design for lifetime maximization in interference-limited wireless sensor networks," IEEE Trans. Wireless Commun., vol. 5, no. 11, pp. 3142-3152, Nov. 2006

[9] H. Liu, M. Bolic, A. Nayak, and I. Stojmenovic, "Taxonomy and challenges of the integration of RFID and wireless sensor networks," IEEE Netw., vol. 22, no. 6, pp. 26-35, Nov./Dec. 2008.

[10] Y. Chen and W. L. Woo, "On modeling of cognitive interrogator-sensor network: Layered discrete memoryless channel and finite-state Markov channel," in Proc. 2nd Int. Workshop CIP, Elba Island, Italy, Jun. 2010, pp. 11-16.

[11] M. Philipose, J. R. Smith, B. Jiang, A. Mamishev, S. Roy, and K. Sundara Rajan, "Battery-free wireless identification and sensing," IEEE Pervasive Comput., vol. 4, no. 1, pp. 37-45, Jan.-Mar. 2005.

[12] G. Marrocco, L. Mattioni, and C. Calabrese, "Multiport sensor RFIDs for wireless passive sensing of objects—Basic theory and early results," IEEE Trans. Antennas Propag., vol. 56, no. 8, pp. 2691-2702, Aug. 2008.

[13] S. Mandal, L. Turicchia, and R. Sarpeshkar, "A low-power, battery-free tag for body sensor networks," IEEE Pervasive Comput., vol. 9, no. 1, pp. 71-77, Jan.-Mar. 2010.

[14] C. M. Kruesi, R. J. Vyas, and M. M. Tentzeris, "Design and development of a novel 3-D cubic antenna for wireless sensor networks (WSNs) and RFID applications," IEEE Trans. Antennas Propag., vol. 57, no. 10, pp. 3293-3299, Oct. 2009

[15] A. Vaz, A. Ubarretxena, I. Zalbide, D. Pardo, H. Solar, A. Garcia-Alonso, and R. Berenguer, "Full passive UHF tag with a temperature sensor suitable for human body temperature monitoring," IEEE Trans. Circuits Syst. II, Exp. Briefs, vol. 57, no. 2, pp. 95-99, Feb. 2010.

[16] C. Occhiuzzi and G. Marrocco, "The RFID technology for neurosciences: Feasibility of limbs' monitoring in sleep diseases," IEEE Trans. Inf. Technol. Biomed., vol. 14, no. 1, pp. 37-43, Jan. 2010.

[17] S. Shrestha, M. Balachandran, M. Agarwal, V. V. Phoha, and K. Varahramyan, "A chipless RFID sensor system for cyber centric monitoring applications," IEEE Trans. Microw. Theory Tech., vol. 57, no. 5, pp. 1303-1309, May 2009.

[18] R. J. M. Vullers, R. V. Schaijk, H. J. Visser, J. Penders, and C. V. Hoof, "Energy harvesting for autonomous wireless sensor networks," IEEE Solid-State Circuits Mag., vol. 2, no. 2, pp. 29-38, Spring 2010.
[19] S. Cheng, K. Tom, L. Thomas, and M. Pecht, "A wireless sensor system for prognostics and health management," IEEE Sensors J., vol. 10, no. 4, pp. 856-862, Apr. 2010.

[20] H. Ramamurthy, B. S. Prabhu, R. Gadh, and A. M. Madni, "Wireless industrial monitoring and control using a smart sensor platform," IEEE Sensors J., vol. 7, no. 5, pp. 611-618, May 2007.

[21] F. Hu, Y. Xiao, and Q. Hao, "Congestion-aware, loss-resilient biomonitoring sensor networking for mobile health applications," IEEE $J$. Sel. Areas Commun., vol. 27, no. 4, pp. 450-465, May 2009.

[22] P.-Y. Chen, W.-T. Chen, Y.-C. Tseng, and C.-F. Huang, "Providing group tour guide by RFIDs and wireless sensor networks," IEEE Trans. Wireless Commun., vol. 8, no. 6, pp. 3059-3067, Jun. 2009.

[23] J. Cho, Y. Shim, T. Kwon, Y. Choi, S. Pack, and S. Kim, "SARIF: A novel framework for integrating wireless sensor and RFID networks," IEEE Wireless Commun., vol. 14, no. 6, pp. 50-56, Dec. 2007.

[24] C.-X. Wang, H.-H. Chen, X. Hong, and M. Guizani, "Cognitive radio network management: Tuning into real-time conditions," IEEE Veh. Technol. Mag., vol. 3, no. 1, pp. 28-35, Mar. 2008.

[25] C.-X. Wang, X. Hong, H.-H. Chen, and J. S. Thompson, "On capacity of cognitive radio networks with average interference power constraints," IEEE Trans. Wireless Commun., vol. 8, no. 4, pp. 1620-1625, Apr. 2009.

[26] P. Sadeghi, R. A. Kennedy, P. B. Rapajic, and R. Shams, "Finite-state Markov modeling of fading channels," IEEE Signal Process. Mag., vol. 25, no. 5, pp. 57-80, Sep. 2008.

[27] H. S. Wang and N. Moayeri, "Finite-state Markov channel-A useful model for radio communication channels," IEEE Trans. Veh. Technol., vol. 44, no. 1, pp. 163-171, Feb. 1995.

[28] C.-X. Wang and W. Xu, "A new class of generative models for burst error characterization in digital wireless channels," IEEE Trans. Commun., vol. 55, no. 3, pp. 453-462, Mar. 2007.

[29] J. N. Laneman, D. N. C. Tse, and G. W. Wornell, "Cooperative diversity in wireless networks: Efficient protocols and outage behavior," IEEE Trans. Inf. Theory, vol. 50, no. 12, pp. 3062-3080, Dec. 2004.

[30] C. S. Patel, G. L. Stüber, and T. G. Pratt, "Statistical properties of amplify and forward relay fading channels," IEEE Trans. Veh. Technol., vol. 55, no. 1, pp. 1-9, Jan. 2006.

[31] R. U. Nabar, H. Boelcskei, and F. W. Kneubhueler, "Fading relay channels: Performance limits and space-time signal design," IEEE J. Sel. Areas Commun., vol. 22, no. 6, pp. 1099-1109, Aug. 2004.

[32] C.-X. Wang, X. Hong, X. Ge, X. Cheng, G. Zhang, and J. S. Thompson, "Cooperative MIMO channel models: A survey," IEEE Commun. Mag., vol. 48, no. 2, pp. 80-87, Feb. 2010.

[33] C. C. Y. Poon, Y. T. Zhang, and S. D. Bao, "A novel biometrics method to secure wireless body area sensor networks for telemedicine and m-health," IEEE Commun. Mag., vol. 44, no. 4, pp. 73-81, Apr. 2006.

[34] R. G. Gallager, Principles of Digital Communication. Cambridge, U.K.: Cambridge Univ. Press, 2008.

[35] J.-F. Chamberland and V. V. Veeravalli, "Decentralized detection in sensor networks," IEEE Trans. Signal Process., vol. 51, no. 2, pp. 407-416, Feb. 2003.

[36] A. Leshem, O. Naparstek, and A. Nehorai, "Information theoretic adaptive radar waveform design for multiple extended targets," IEEE J. Sel. Topics Signal Process., vol. 1, no. 1, pp. 42-55, Jun. 2007.

[37] A. Ishimaru, Wave Propagation and Scattering in Random Media. Oxford, U.K.: Oxford Univ. Press, 1997.

[38] T. M. Cover and J. A. Thomas, Elements of Information Theory. New York: Wiley, 1991.

[39] R. G. Gallager, Information Theory and Reliable Communications. New York: Wiley, 1968.

[40] Y. Chen and P. Rapajic, "Decentralized wireless relay network channel modeling: An analogous approach to mobile radio channel characterization," IEEE Trans. Commun., vol. 58, no. 2, pp. 467-473, Feb. 2010.

[41] A. F. Molisch, Wireless Communications. Chichester, U.K.: Wiley, 2005.

[42] S. R. Saunders and A. Aragon-Zavala, Antennas and Propagation for Wireless Communication Systems. Chichester, U.K.: Wiley, 2007.

[43] T. Q. S. Quek, D. Dardari, and M. Z. Win, "Energy efficiency of dense wireless sensor networks: To cooperate or not to cooperate," IEEE J. Sel. Areas Commun., vol. 25, no. 2, pp. 459-470, Feb. 2007.

[44] M. Patzold and K. Yang, "An exact solution for the level-crossing rate of shadow fading processes modelled by using the sum-of-sinusoids principle," Wireless Pers. Commun., vol. 52, no. 1, pp. 57-68, Jan. 2010.

[45] R. Janaswamy, "Angle and time of arrival statistics for the Gaussian scatter density model," IEEE Trans. Wireless Commun., vol. 1, no. 3, pp. 488-497, Jul. 2002. 


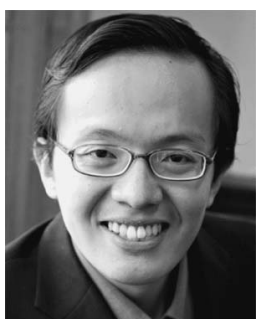

Yifan Chen (M'06) received the B.Eng. (Hons. I) and $\mathrm{Ph} . \mathrm{D}$. degrees in electrical and electronic engineering from Nanyang Technological University (NTU), Singapore, in 2002 and 2006, respectively.

From 2005 to 2007, he was a Research Fellow with the Singapore-University of Washington Alliance in Bioengineering, NTU. From 2007 to 2010, he was a Senior Lecturer with the School of Engineering, University of Greenwich, London, U.K. He is currently a Lecturer with the School of Electrical, Electronic, and Computer Engineering, Newcastle University, Newcastle upon Tyne, U.K. He is also an Adjunct Associate Professor with the School of Computer, Electronics, and Information, Guangxi University, Nanning, China. His current research interests involve wireless and pervasive communications for healthcare, microwave biomedical imaging, wireless channel modeling, wireless communication theory, and cognitive dynamic systems.

Dr. Chen received the Promising Research Fellowship in 2010 and the Early Career Research Excellence Award in 2009 from the University of Greenwich in recognition of his exceptional research contributions and potential.

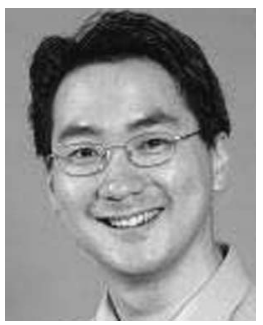

Wai Lok Woo was born in Malaysia. He received the B.Eng. degree (First-Class Hons.) in electrical and electronics engineering and the Ph.D. degree from Newcastle University, Newcastle upon Tyne, U.K.

$\mathrm{He}$ is currently a Senior Lecturer with the School of Electrical, Electronic, and Computer Engineering, Newcastle University. He currently serves on the editorial boards of many international signal processing journals. He actively participates in international conferences and workshops and serves on their organizing and technical committees. In addition, he acts as a Consultant to a number of industrial companies that involve the use of statistical signal processing techniques. His major research is in mathematical theory and algorithms for nonlinear signal processing. This includes areas of blind signal separation, signal characterization and identification, informationtheoretic learning, deconvolution, and restoration.

Dr. Woo received the IEE Prize and the British Scholarship in 1998 to continue his research.

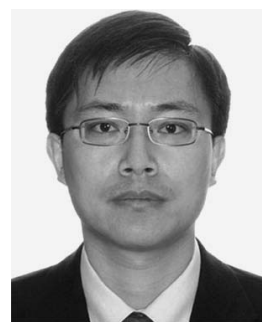

Cheng-Xiang Wang (S'01-M'05-SM'08) received the B.Sc. and M.Eng. degrees in communication and information systems from Shandong University, Jinan, China, in 1997 and 2000, respectively, and the $\mathrm{Ph} . \mathrm{D}$. degree in wireless communications from Aalborg University, Aalborg, Denmark, in 2004.

He was a Research Assistant with the Technical University of Hamburg-Harburg, Hamburg, Germany, from 2000 to 2001, a Research Fellow with the University of Agder, Grimstad, Norway, from 2001 to 2005, and a Visiting Researcher with Siemens AG-Mobile Phones, Munich, Germany, in 2004. He is an Honorary Fellow of the University of Edinburgh, Edinburgh, U.K., a Chair Professor of Shandong University, a Guest Professor of the Huazhong University of Science and Technology, Wuhan, China, an Adjunct Professor of Guilin University of Electronic Technology, Guilin, China, and a Guest Researcher of Xidian University, Xi' an, China. He has been with Heriot-Watt University, Edinburgh, since 2005, first as a Lecturer and then as a Reader in 2009. He is leading several projects funded by the Engineering and Physical Sciences Research Council (EPSRC), Mobile VCE, and industries, including the Research Councils UK funded "U.K.-China Science Bridges: R\&D on (B)4G Wireless Mobile Communications." He has published one book chapter and about 140 papers in refereed journals and conference proceedings. He is currently serving as Editor for Wireless Communications and Mobile Computing Journal (Wiley), the Security and Communication Networks Journal (Wiley), and the Journal of Computer Systems, Networks, and Communications (Hindawi). His current research interests include wireless channel modeling and simulation, cognitive radio networks, vehicular communication networks, green radio communications, cooperative multiple-input-multiple-output, cross-layer design of wireless networks, utra-wideband, and beyond fourth-generation wireless communications.

Dr. Wang is a member of the Institution of Engineering and Technology (IET), a Fellow of the High Education Academy (HEA), and a member of the EPSRC Peer Review College. He served as Editor for the IEEE TRANSACTIONS ON WiRELESS COMMUNICATIONS from 2007 to 2009. He is the leading Guest Editor for the IEEE JOURNAL ON SELECTED AREAS IN COMMUNICATIONS Special Issue on Vehicular Communications and Networks. He served or is serving as Technical Program Committee (TPC) member, TPC Chair, and General Chair for about 60 international conferences. He received the Best Paper Award at the IEEE Global Communications Conference in 2010 . $\mathrm{He}$ is listed in the "Dictionary of International Biography 2008 and 2009," "Who's Who in the World 2008 and 2009," "Great Minds of the 21st Century 2009," and "2009 Man of the Year." 